\title{
Balance of Plant Requirements for a Nuclear Hydrogen Plant
}

\author{
Bradley Ward
}

April 2006

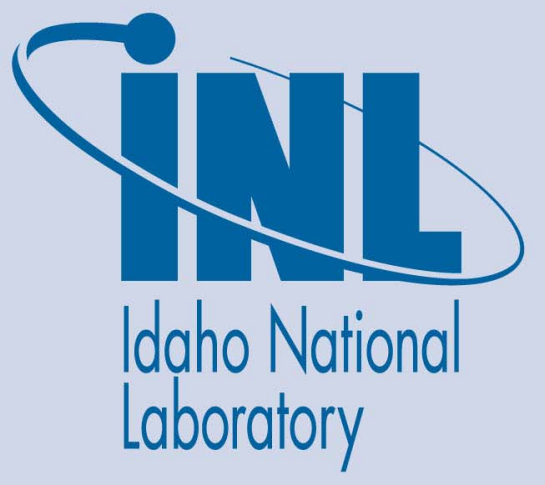

The INL is a U.S. Department of Energy National Laboratory operated by Battelle Energy Alliance 
INL/EXT-06-11232

Rev. 1

\title{
Balance of Plant Requirements for a Nuclear Hydrogen Plant
}

\author{
Bradley Ward
}

April 2006

Idaho National Laboratory
Idaho Falls, Idaho 83415

Prepared for the

U.S. Department of Energy

Office of Nuclear Energy

Under DOE Idaho Operations Office

Contract DE-AC07-05ID14517 


\section{SUMMARY}

This document describes the requirements for the components and systems that support the hydrogen production portion of a 600 megawatt thermal (MWt) Next Generation Nuclear Plant (NGNP). These systems, defined as the "balance-of-plant" (BOP), are essential to operate an effective hydrogen production plant. Examples of BOP items are: heat recovery and heat rejection equipment, process material transport systems (pumps, valves, piping, etc.), control systems, safety systems, waste collection and disposal systems, maintenance and repair equipment, heating, ventilation, and air conditioning (HVAC), electrical supply and distribution, and others.

The requirements in this document are applicable to the two hydrogen production processes currently under consideration in the DOE Nuclear Hydrogen Initiative. These processes are the sulfur iodide (S-I) process and the high temperature electrolysis (HTE) process. At present, the other two hydrogen production process - the hybrid sulfur-iodide electrolytic process (SE) and the calcium-bromide process $(\mathrm{Ca}-\mathrm{Br})$ - are under flow sheet development and not included in this report. While some features of the balance-of-plant requirements are common to all hydrogen production processes, some details will apply only to the specific needs of individual processes. 


\section{CONTENTS}

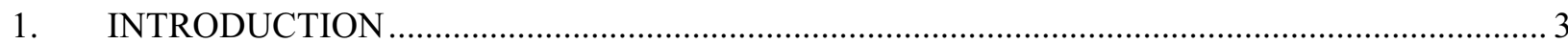

2. CANDIDATE HYDROGEN PRODUCTION PROCESSES …...................................................... 6

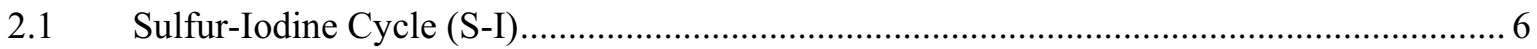

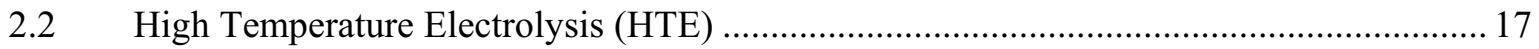

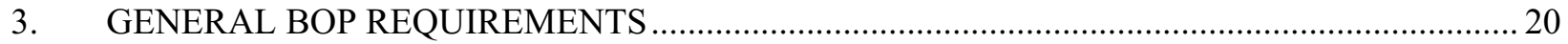

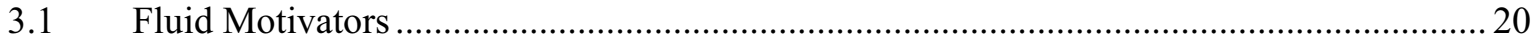

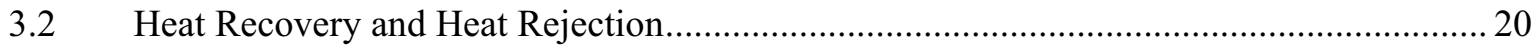

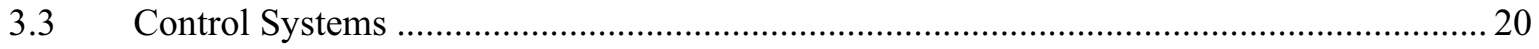

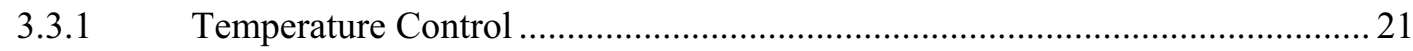

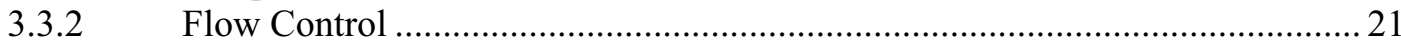

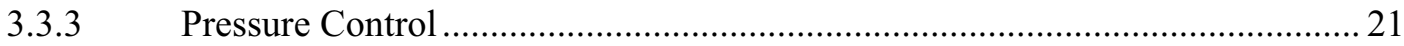

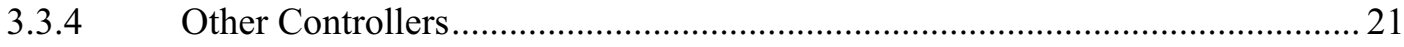

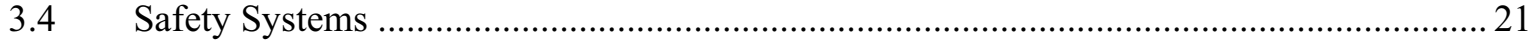

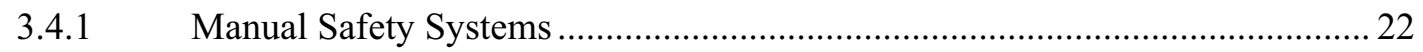

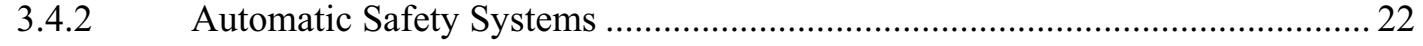

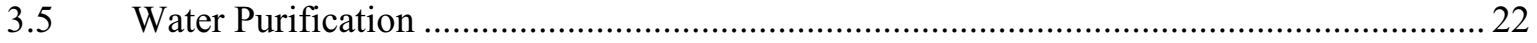

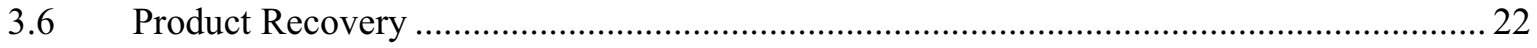

3.7 Non-Product Stream Treatment and Disposal .......................................................... 22

3.8 Heating, Ventilation, and Air Conditioning (HVAC) …............................................... 23

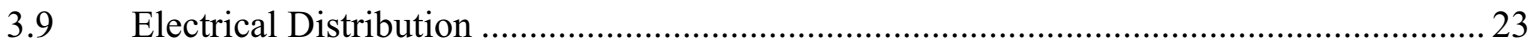

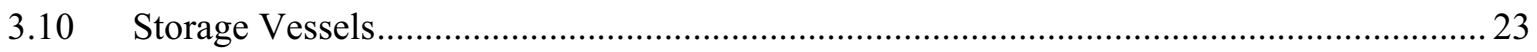

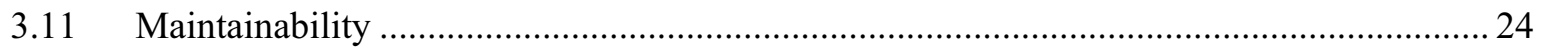

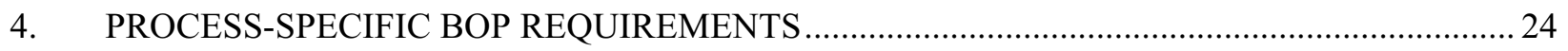

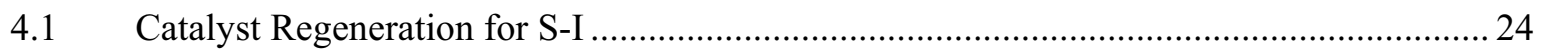

4.2 Corrosion Product Removal in S-I Process .................................................................. 24

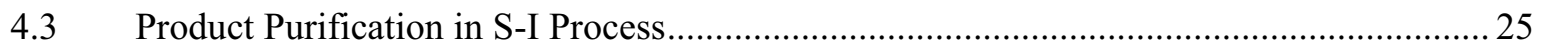

4.4 Hydrogen Purification in the HTE Process .................................................................... 25

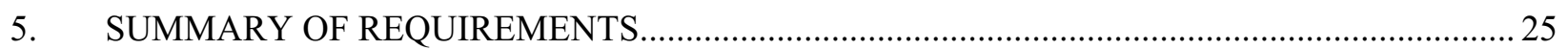

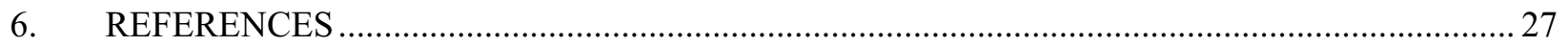




\section{FIGURES}

Figure 1. Schematic of Next Generation Nuclear Plant .......................................................................... 4

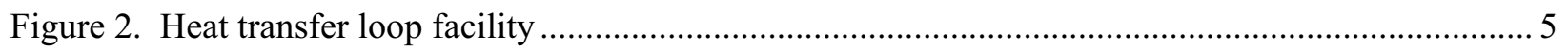

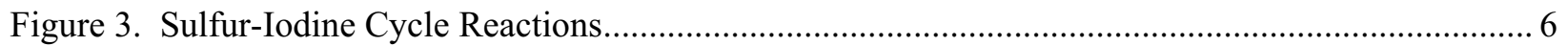

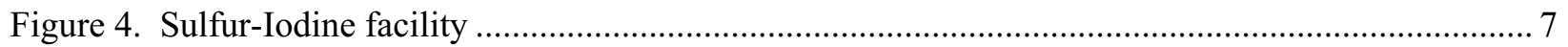

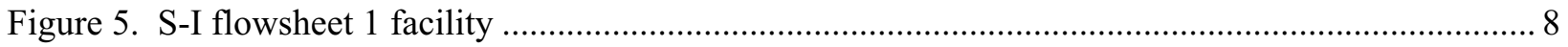

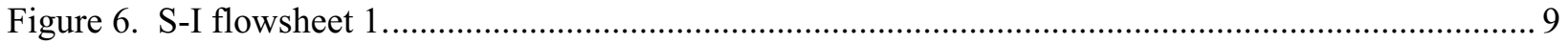

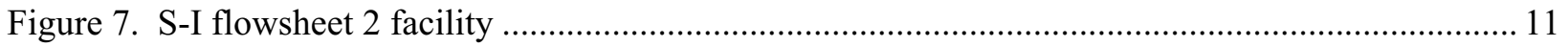

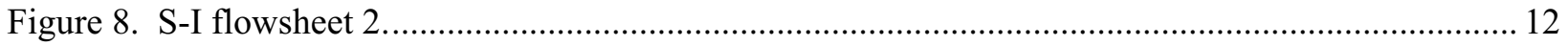

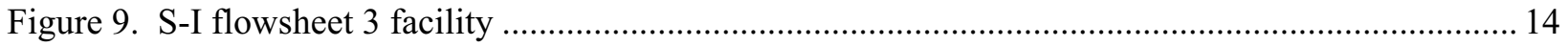

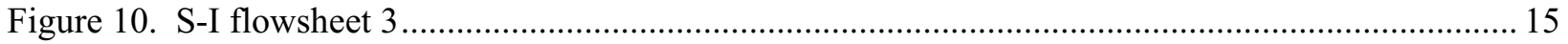

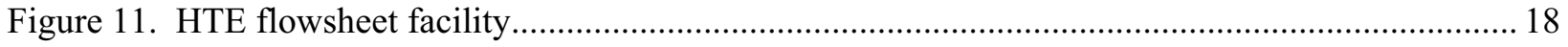

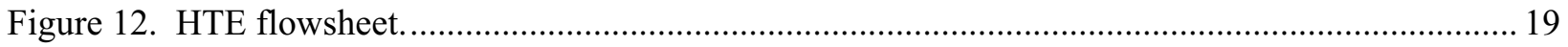

TABLES

Table 1. Heat transfer loop equipment footprints ............................................................................ 5

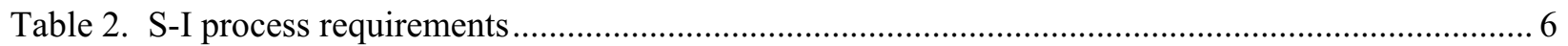

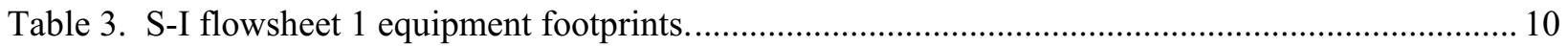

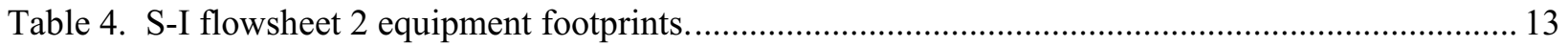

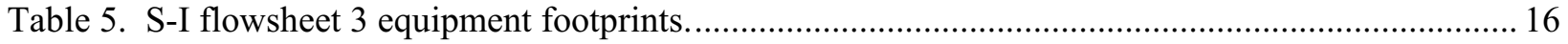

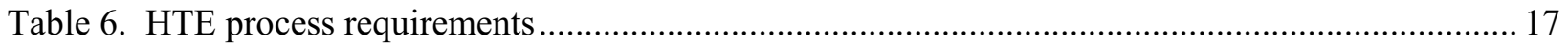

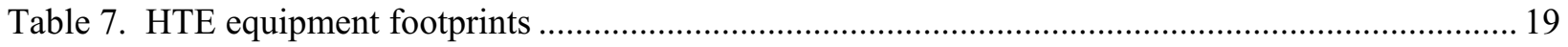

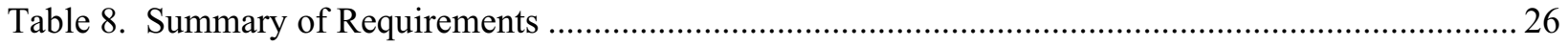




\section{Balance of Plant Requirements for a Nuclear Hydrogen Plant}

\section{INTRODUCTION}

This document describes high-level functional requirements for the hydrogen production plant "balance of plant," or BOP. The BOP is the sum of systems and equipment that support the main chemical reactors performing the hydrogen production reactions in the plant. BOP is a term commonly used in the electrical power industry to describe the sum of equipment surrounding a coal burner, a nuclear reactor, or other such power generator. It is less commonly used in the chemical industry, but in the context of the Department of Energy (DOE) Nuclear Hydrogen Initiative (NHI), it is a useful term to apply to the hydrogen production plant, since the BOP for hydrogen production will be developed in parallel with basic research into hydrogen production methods.

The hydrogen production plant, along with its BOP components, will be part of a 600 megawatt thermal (MWt) Next Generation Nuclear Plant (NGNP). The NGNP, as envisioned by the DOE Office of Nuclear Energy (NE), is the combination of two technological concepts: a Generation IV Very High Temperature Gas-Cooled Reactor (VHTR) and an advanced hydrogen production plant. The NGNP will be capable of producing both electricity and hydrogen at costs that are economically competitive with other more established production methods. The goal of NGNP is to provide a means of meeting domestic energy needs without relying on the use of fossil fuels and without releasing any greenhouse gases.

A notional schematic of the NGNP is shown in Figure 1. The VHTR provides nuclear process heat to the hydrogen production plant through a high temperature heat transfer loop. The VHTR also provides electricity to operate the hydrogen plant and to power key hydrogen production steps. Various supporting systems in the hydrogen production plant are required for fluid handling, storage, and process support. Within DOE NE, research on the VHTR is being accomplished under the Generation IV Program (Gen IV), and research on nuclear hydrogen production systems and the reactor/process interface is being conducted under the Nuclear Hydrogen Initiative (NHI).

This document focuses on the requirements for the hydrogen production plant BOP. In Figure 1, the hydrogen production plant is shown in yellow. BOP, aside from the chemical reactors used to produce hydrogen, is needed to operate and maintain the hydrogen production reactors and to manage the flow of energy and materials into and out of hydrogen plant components.

This document describes the BOP requirements that apply in general to all candidate hydrogen production processes that are under consideration for the NGNP. It is the first revision of "Balance of Plant for a Requirements for a Nuclear Hydrogen Pilot Plant," ANL W7500-0003-ES-00. These candidate processes are the sulfur-iodine (SI) cycle and the high temperature electrolysis (HTE) process. All of these processes will require support systems, or BOP, to perform their hydrogen production steps. Examples of BOP items are heat recovery and heat rejection equipment; process material transport systems (pumps, valves, piping, etc.); control systems; safety systems; waste collection and disposal systems; maintenance and repair equipment; heating, ventilation, and air conditioning (HVAC) equipment; electrical supply and distribution systems; chemical storage equipment; and equipment associated with start-up and shutdown operations.

The requirements presented here are general in nature and provide, for the most part, a listing of necessary equipment. Detailed descriptions of support systems for each candidate process, along with quantitative sizing information, will not be provided, since such information cannot be developed until all process details have been determined and complete flow sheet models have been devised. 


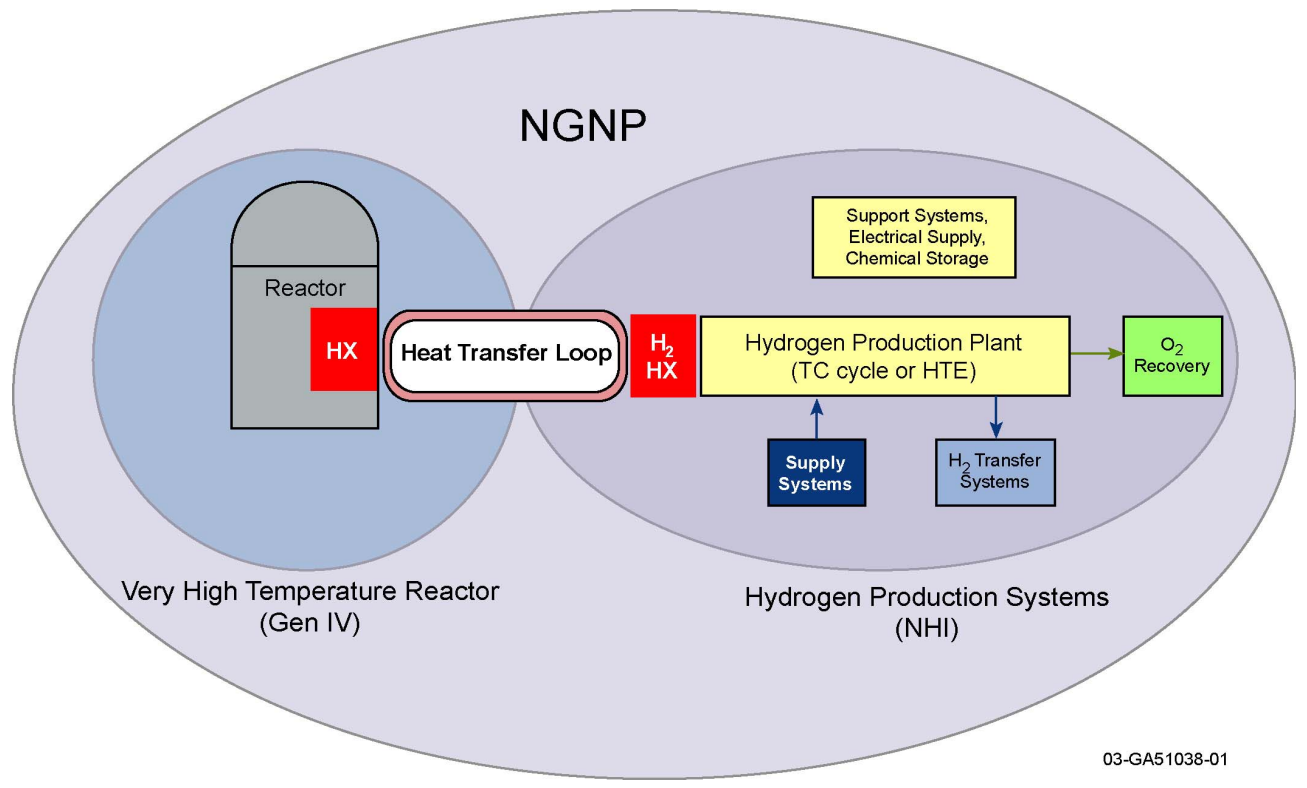

Figure 1. Schematic of Next Generation Nuclear Plant

The heat transfer loop consists of multiple compressors, turbines, heat exchangers. Heat transfer loop process requirements were estimated utilizing the HYSYS model reported by Carl Stoots, James O'Brien, Mike McKellar, Grant Hawkes, and Stephen Herring in an internal INEEL report published in June 2004. 


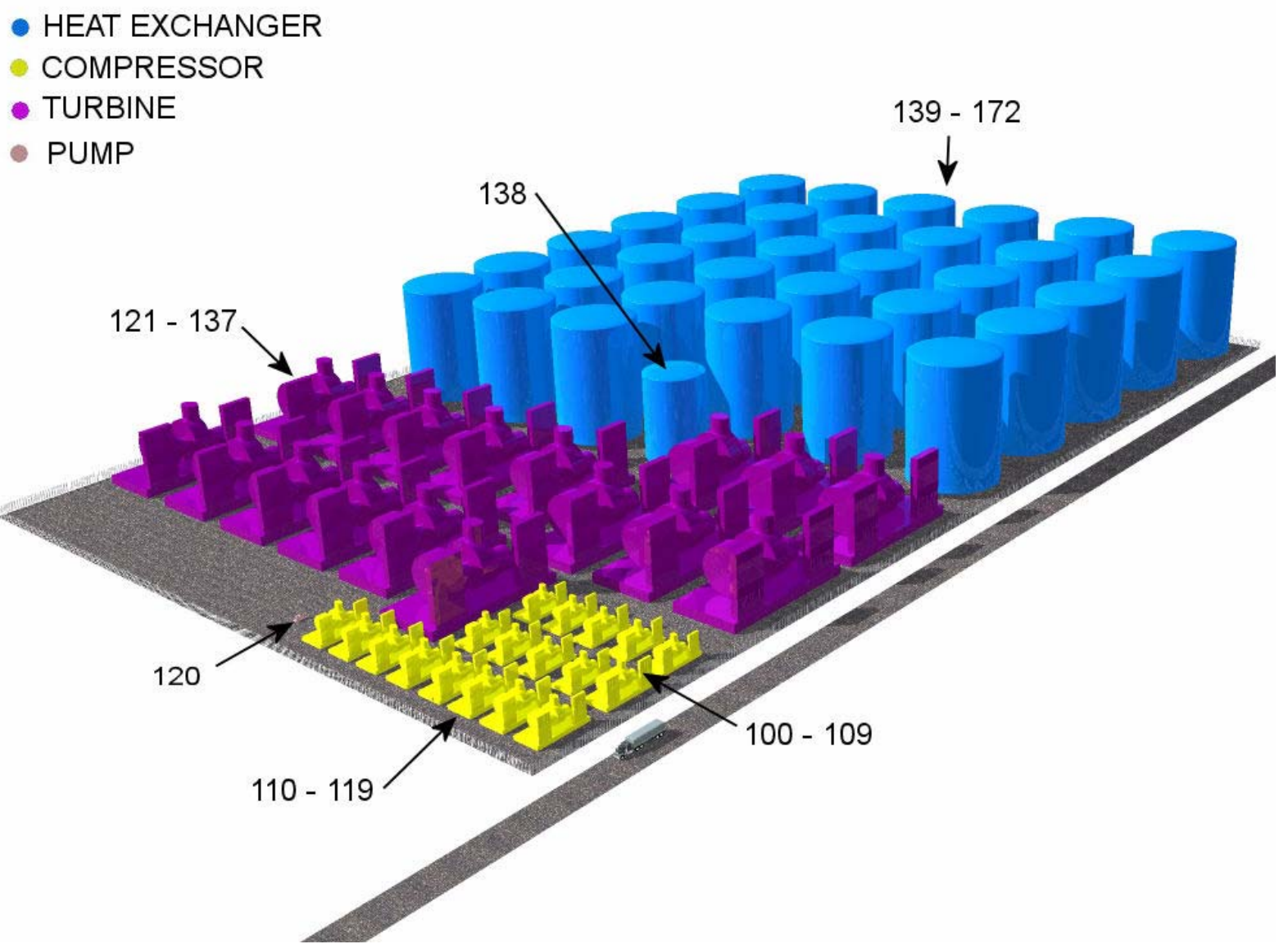

Figure 2. Heat transfer loop facility

Table 1. Heat transfer loop equipment footprints

\begin{tabular}{|l|l|l|c|r|}
\hline Heat Transfer Loop & \multicolumn{1}{|c|}{ Item } & \multicolumn{1}{|c|}{ Equipment Description } & Area $\left(\mathbf{f t}^{\mathbf{2}}\right)$ & Vol $\left(\mathbf{f t}^{\mathbf{3}}\right)$ \\
\hline Compressors & $100-119$ & Low Pressure Compressor, 13200 hp, Ni Alloy, 20 Required & 1657.3 & 47736.1 \\
\hline & 120 & Compressor, 2980 hp, Ni Alloy & 62.1 & 345.9 \\
\hline Turbines & $121-137$ & Steam Turbine, 40235 hp, Ni Alloy, 17 Required & 11312.4 & 851327.0 \\
\hline Heat Exchangers & 138 & $\begin{array}{l}\text { Recuperator, CS/SiC Shell and tube, fixed tube sheet heat } \\
\text { exchanger }\end{array}$ & 3879.0 & 409012.0 \\
\hline & $139-172$ & $\begin{array}{l}\text { Recuperator, CS/SiC Shell and tube, fixed tube sheet heat } \\
\text { exchanger, 34 Required }\end{array}$ & 7610.7 & 1124064.7 \\
\hline & & Total for Equipment & $\mathbf{4 8 8 , 1 6 1}$ & $\mathbf{5 4 , 0 5 4 , 8 3 9}$ \\
\hline
\end{tabular}

This document begins with an overview of candidate hydrogen production processes. General BOP requirements are then given and described. After the general BOP requirements, requirements specific to individual processes are given. It is intended that the requirements presented here will be considered by process designers, modelers, and cost estimators, so that complete plant designs can be generated and fairly evaluated in terms of function and cost. 


\section{CANDIDATE HYDROGEN PRODUCTION PROCESSES}

Currently, there are four hydrogen production processes that are receiving the most attention by researchers within the DOE Nuclear Hydrogen Initiative. These processes are the sulfur-iodine cycle (S-I) and High Temperature Electrolysis (HTE). These hydrogen production processes are briefly described below. For more complete process descriptions, see INL/EXT-04-01791, Infrastructure Requirements for a Nuclear Hydrogen Pilot Plant.

\subsection{Sulfur-lodine Cycle (S-I)}

The sulfur-iodine cycle consists of three chemical reactions. In the first reaction, known as the Bunsen Reaction, sulfur dioxide $\left(\mathrm{SO}_{2}\right)$, iodine $\left(\mathrm{I}_{2}\right)$, and water $\left(\mathrm{H}_{2} \mathrm{O}\right)$ react at low temperatures (approximately $120^{\circ} \mathrm{C}$ ) to form sulfuric acid $\left(\mathrm{H}_{2} \mathrm{SO}_{4}\right)$ and hydrogen iodide $(\mathrm{HI})$. In the second reaction, $\mathrm{H}_{2} \mathrm{SO}_{4}$ is decomposed over a catalyst at $850{ }^{\circ} \mathrm{C}$ to form $\mathrm{SO}_{2}, \mathrm{H}_{2} \mathrm{O}$, and oxygen $\left(\mathrm{O}_{2}\right)$. In the third reaction, $\mathrm{HI}$ is decomposed into hydrogen $\left(\mathrm{H}_{2}\right)$ and $\mathrm{I}_{2}$ at approximately $300{ }^{\circ} \mathrm{C}$. These reactions are shown in Figure 3.

$$
\begin{array}{lr}
\mathrm{SO}_{2}+\mathrm{I}_{2}+2 \mathrm{H}_{2} \mathrm{O} \rightarrow \mathrm{H}_{2} \mathrm{SO}_{4}+2 \mathrm{HI} & \text { lowtemperature reaction } \\
\mathrm{H}_{2} \mathrm{SO}_{4} \rightarrow \mathrm{SO}_{2}+\mathrm{H}_{2} \mathrm{O}+\frac{1}{2} \mathrm{O}_{2} & \text { decomposition at } 850{ }^{\circ} \mathrm{C} \\
2 \mathrm{HI} \rightarrow \mathrm{H}_{2}+\mathrm{I}_{2} & \text { decomposition at } \approx 300{ }^{\circ} \mathrm{C}
\end{array}
$$

Figure 3. Sulfur-Iodine Cycle Reactions

When the process reaches steady state, the inputs are $\mathrm{H}_{2} \mathrm{O}$ and high-temperature thermal energy, and the outputs are $\mathrm{H}_{2}, \mathrm{O}_{2}$, and lower temperature thermal energy. The chemicals $\mathrm{H}_{2} \mathrm{SO}_{4}, \mathrm{SO}_{2}, \mathrm{HI}$, and $\mathrm{I}_{2}$, are recycled in the process by formation and decomposition mechanisms. The S-I thermochemical process consists of 3 chemical processing sections and process support systems. The three processing units are a Main Solution Reaction section (Flowsheet 1), the Sulfuric Acid Concentration and Decomposition section (Flowsheet 2), and a Hydrogen Iodide (HI) Decomposition section (Flowsheet 3). Process requirements were estimated utilizing the process flows identified in General Atomics report GA-

\begin{tabular}{|c|c|}
\hline Sulfur-Iodine Cycle & $600 \mathrm{MW}$ \\
\hline Heating Load (either electrical or fossil) & $576 \mathrm{MW}$ \\
\hline Power for Prime Movers & $24 \mathrm{MW}$ \\
\hline \multicolumn{2}{|l|}{ Process Space Requirement } \\
\hline No. of Equipment & 77 \\
\hline No. of Processing Sections & 3 \\
\hline Vessel Footprint & $48,201 \mathrm{ft}^{2}$ \\
\hline Plant Footprint (based on 10:1 ratio) & $482,010 \mathrm{ft}^{2}$ \\
\hline Access Area (based on 3:4 plant footprint ratio) & $361,508 \mathrm{ft}^{2}$ \\
\hline Control System (based on 1:8 plant footprint ratio) & $60,251 \mathrm{ft}^{2}$ \\
\hline Total Space Requirements & $903,769 \mathrm{ft}^{2}$ \\
\hline
\end{tabular}
A24285, June 2003.

Table 2. S-I process requirements 


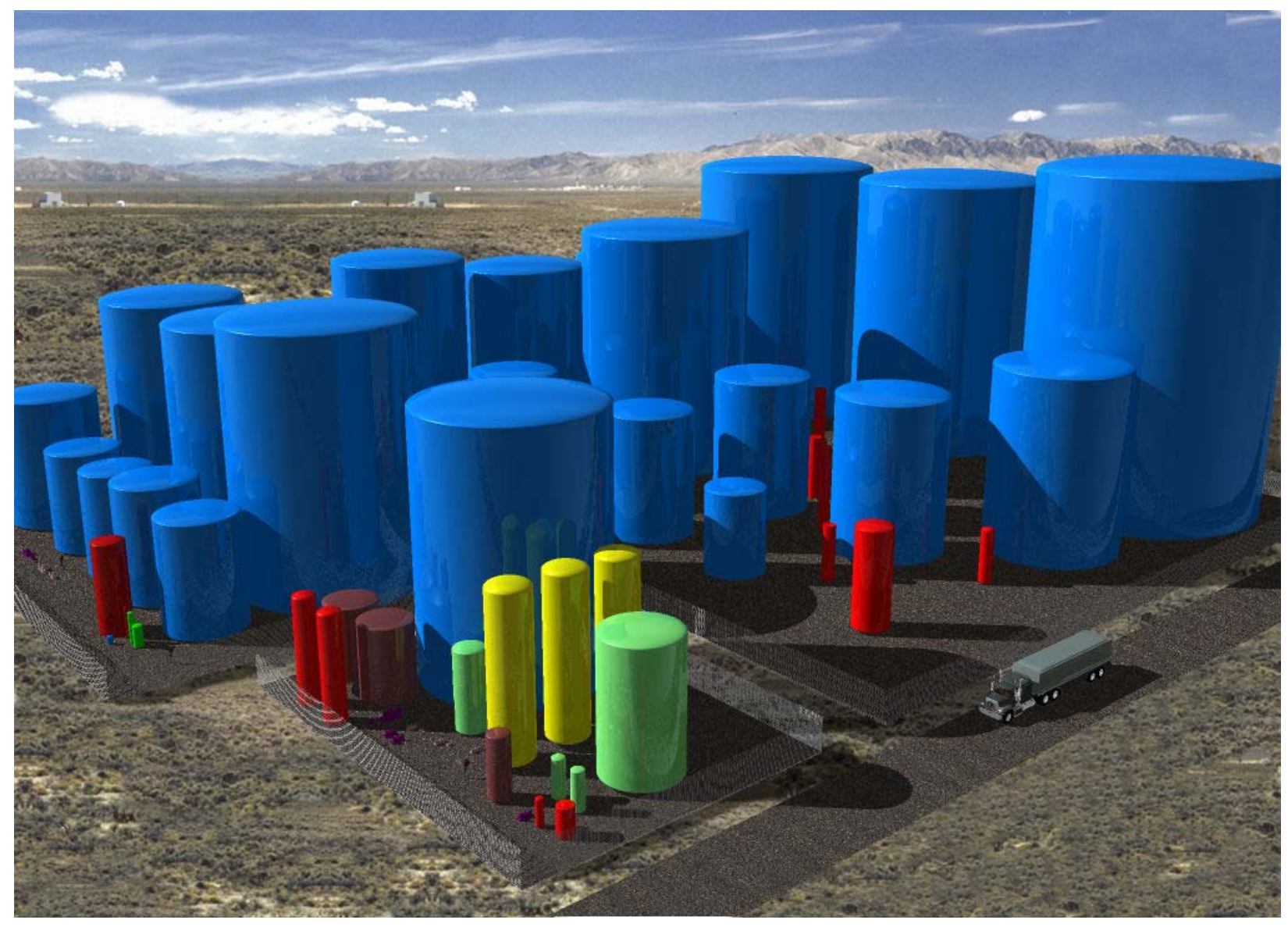

Figure 4. Sulfur-Iodine facility 


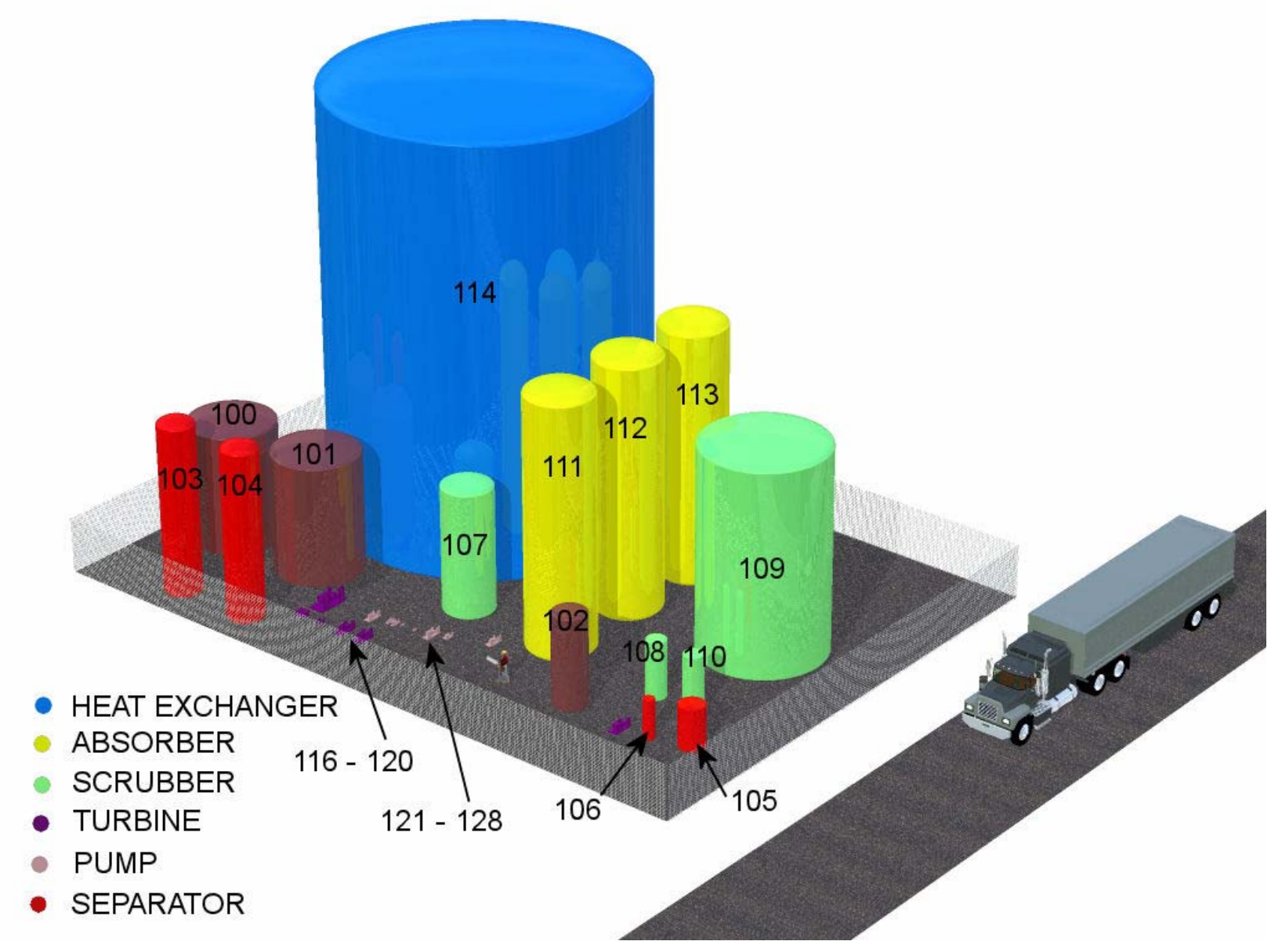

Figure 5. S-I flowsheet 1 facility 


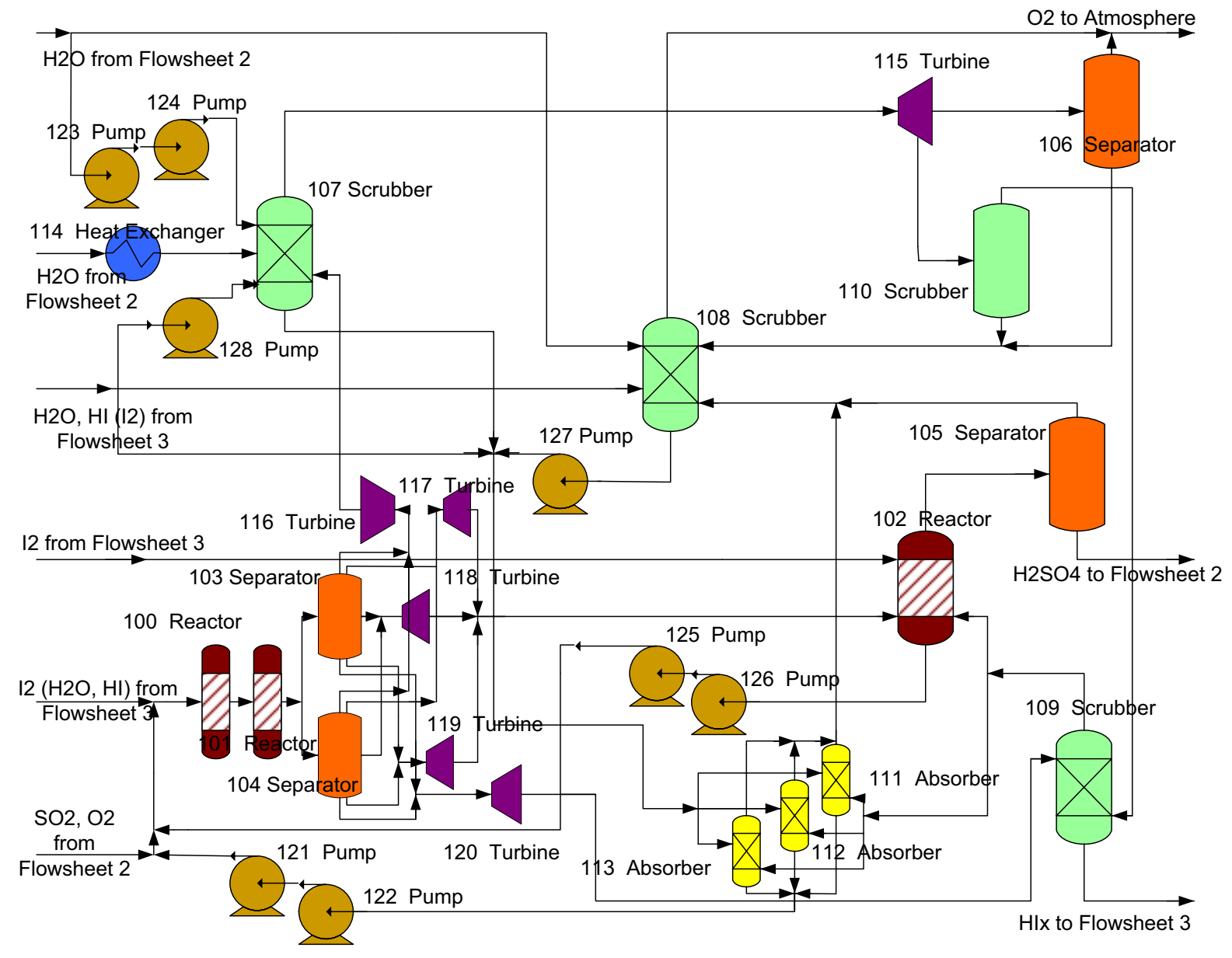

Figure 6. S-I flowsheet 1. 
Table 3. S-I flowsheet 1 equipment footprints.

\begin{tabular}{|c|c|c|c|c|}
\hline Flowsheet 1 & Item & Equipment Description & Area $\left(\mathrm{ft}^{2}\right)$ & Vol $\left(\mathrm{ft}^{3}\right)$ \\
\hline \multirow[t]{3}{*}{ Reactors } & 100 & $\begin{array}{l}\text { Flow reactor w/intergral heat exchanger, Shell and tube, fixed } \\
\text { tube sheet heat exchanger }\end{array}$ & 183.0 & 4191.1 \\
\hline & 101 & $\begin{array}{l}\begin{array}{l}\text { Flow reactor w/intergral heat exchanger, Shell and tube, fixed } \\
\text { tube sheet heat exchanger }\end{array} \\
\end{array}$ & 183.0 & 4191.1 \\
\hline & 102 & $\begin{array}{l}\mathrm{H}_{2} \mathrm{SO}_{4} \text { boost reactor, Vertical vessel, } 6 \mathrm{ft} \text { dia. } X 17.5 \mathrm{ft} \text { tall, } \\
\text { Carbon Steel }\end{array}$ & 28.3 & 494.6 \\
\hline \multirow[t]{4}{*}{ Separators } & 103 & $\begin{array}{l}\text { 3-Phase Cyclonic knockout drum, Vertical Vessel, } 6.5 \mathrm{ft} \text { dia. X } \\
32.5 \mathrm{ft} \text { tall, Carbon Steel }\end{array}$ & 33.0 & 1078.0 \\
\hline & 104 & $\begin{array}{l}\text { 3-Phase Cyclonic knockout drum, Vertical Vessel, } 6.5 \mathrm{ft} \text { dia. X } \\
32.5 \mathrm{ft} \text { tall, Carbon Steel }\end{array}$ & 33.0 & 1078.0 \\
\hline & 105 & Flash drum, Vertical Vessel, $4.5 \mathrm{ft}$ dia. $X 8 \mathrm{ft}$ tall, Carbon Steel & 15.9 & 127.2 \\
\hline & 106 & $\begin{array}{l}\text { Secondary } \mathrm{O}_{2} \text { water knockout drum, Horizontal Vessel, } 2 \mathrm{ft} \\
\text { dia. } \times 7.5 \mathrm{ft} \text { long, Carbon Steel }\end{array}$ & 3.1 & 23.6 \\
\hline \multirow[t]{4}{*}{ Scrubbers } & 107 & $\begin{array}{l}\text { Primary O2 scrubber, Vertical Vessel, } 9 \mathrm{ft} \text { dia. } \times 24 \mathrm{ft} \text { tall, } \\
\text { Carbon Steel }\end{array}$ & 63.6 & 1526.0 \\
\hline & 108 & $\begin{array}{l}\text { Secondary O2 Scrubber, Vertical Vessel, } 3.5 \mathrm{ft} \text { dia x } 11 \mathrm{ft} \text { tall, } \\
\text { Carbon Steel }\end{array}$ & 9.6 & 105.8 \\
\hline & 109 & $\begin{array}{l}\text { Lower phase } \mathrm{SO}_{2} \text { scrubber, Vertical Vessel, } 22 \mathrm{ft} \text { dia. } \times 39.5 \mathrm{ft} \\
\text { tall, Carbon Steel }\end{array}$ & 379.9 & 15007.6 \\
\hline & 110 & $\begin{array}{l}\text { Primary O2 water knockout drum, Vertical Vessel, } 3.5 \mathrm{ft} \text { dia. X } \\
11 \mathrm{ft} \text { tall, Carbon Steel }\end{array}$ & 9.6 & 105.8 \\
\hline \multirow[t]{3}{*}{ Absorbers } & 111 & $\begin{array}{l}\mathrm{SO}_{2} \text { absorber, Vertical Vessel, } 12 \mathrm{ft} \text { dia. X } 48 \mathrm{ft} \text { tall, Carbon } \\
\text { Steel }\end{array}$ & 113.0 & 5426.0 \\
\hline & 112 & $\begin{array}{l}\mathrm{SO}_{2} \text { absorber, Vertical Vessel, } 12 \mathrm{ft} \text { dia. X } 48 \mathrm{ft} \text { tall, Carbon } \\
\text { Steel }\end{array}$ & 113.0 & 5426.0 \\
\hline & 113 & $\begin{array}{l}\mathrm{SO}_{2} \text { absorber, Vertical Vessel, } 12 \mathrm{ft} \text { dia. X } 48 \mathrm{ft} \text { tall, Carbon } \\
\text { Steel }\end{array}$ & 113.0 & 5426.0 \\
\hline Heat Exchangers & 114 & Shell and tube, fixed tube sheet exchanger, $\mathrm{CS} / \mathrm{Cu}$ & 2427.0 & 202423.6 \\
\hline \multirow[t]{6}{*}{ Turbines } & 115 & $\mathrm{O}_{2}$ Axial Gas Turbine, 1497 shaft hp, Ni Alloy & 15.6 & 44.3 \\
\hline & 116 & Liquid Expander, 34 shaft hp, Carbon steel & 1.8 & 2.2 \\
\hline & 117 & Overhead Axial Gas Turbine Expander, 27 shaft hp, Ni Alloy & 1.8 & 2.2 \\
\hline & 118 & Side Liquid Expander, 35.8 shaft hp, Ni Alloy & 1.8 & 2.2 \\
\hline & 119 & Bottoms Liquid Expander, 127.1 shaft hp, Ni Alloy & 2.4 & 3.0 \\
\hline & 120 & Bottoms Liquid Expander, 127.1 shaft hp, Ni Alloy & 2.4 & 3.0 \\
\hline \multirow[t]{9}{*}{ Pumps } & 121 & Fluorocarbon lined centrifugal pump, 31.8 shaft hp & 1.5 & 1.1 \\
\hline & 122 & Fluorocarbon lined centrifugal pump, 31.8 shaft $\mathrm{hp}$ & 1.5 & 1.1 \\
\hline & 123 & Fluorocarbon lined centrifugal pump, 7.3 shaft hp & 1.3 & 0.9 \\
\hline & 124 & Fluorocarbon lined centrifugal pump, 7.3 shaft $\mathrm{hp}$ & 1.3 & 0.9 \\
\hline & 125 & $\begin{array}{l}\text { Boost Reactor Centrifugal Pump, } 4.4 \text { shaft hp, Fluorocarbon } \\
\text { Lined }\end{array}$ & 1.3 & 0.9 \\
\hline & 126 & $\begin{array}{l}\text { Boost Reactor Centrifugal Pump, } 4.4 \text { shaft hp, Fluorocarbon } \\
\text { Lined }\end{array}$ & 1.3 & 0.9 \\
\hline & 127 & Fluorocarbon lined Centrifugal pump, 2.2 shaft hp & 1.3 & 0.9 \\
\hline & 128 & Fluorocarbon lined centrifugal pump, 7.3 shaft $\mathrm{hp}$ & 1.3 & 0.9 \\
\hline & & Total for Equipment & 3,266 & 5,511 \\
\hline
\end{tabular}




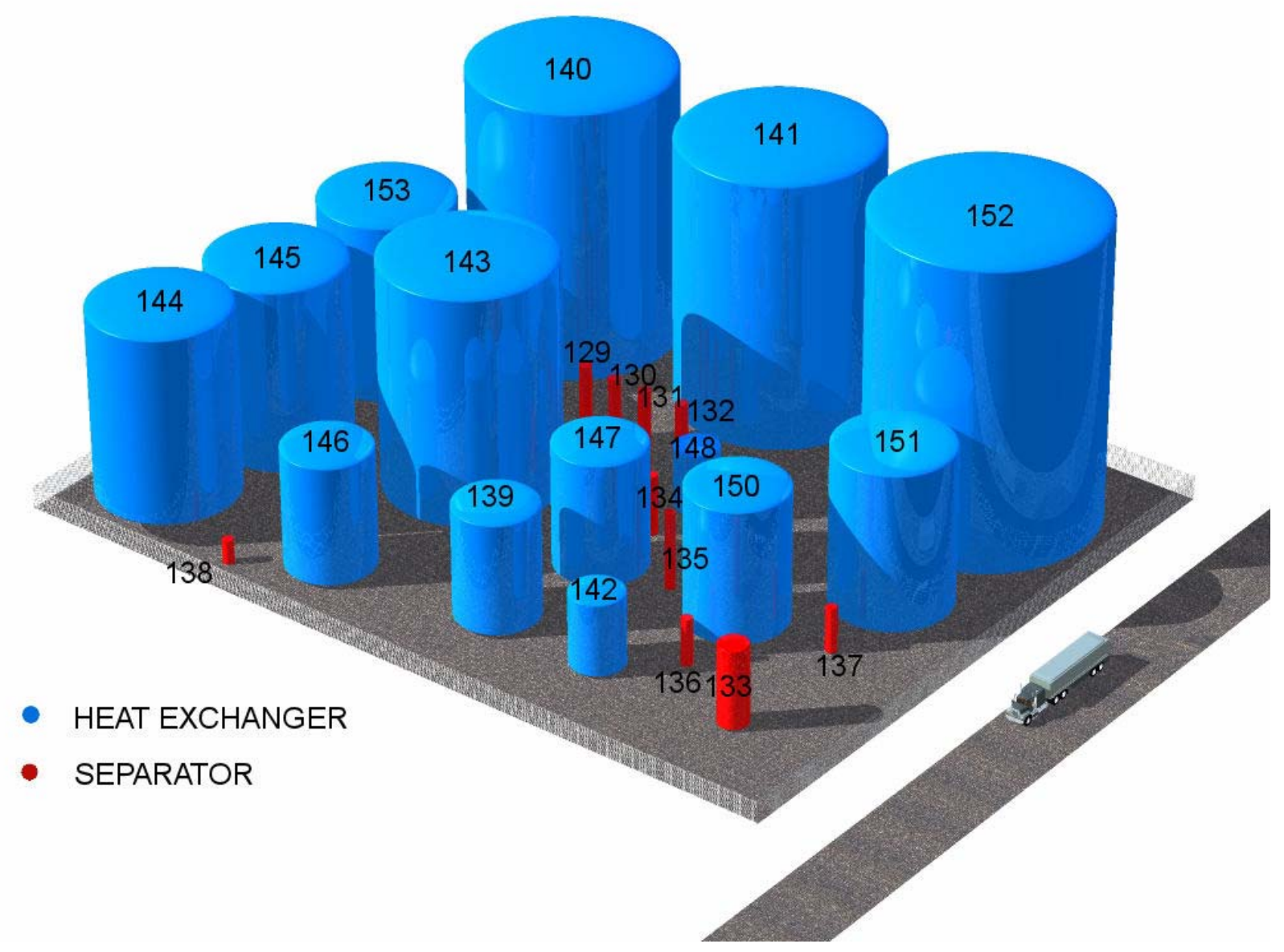

Figure 7. S-I flowsheet 2 facility 


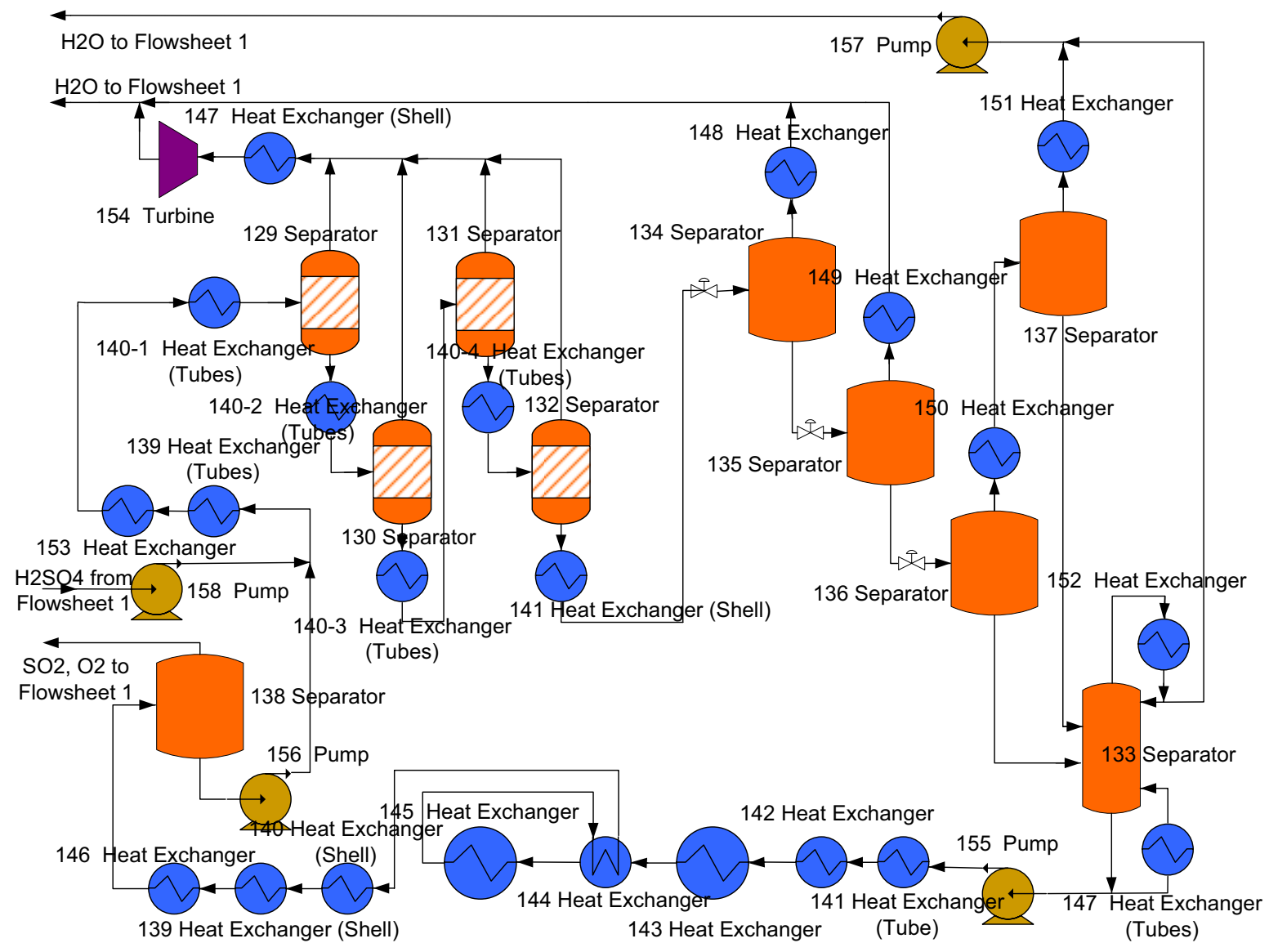

Figure 8. S-I flowsheet 2. 
Table 4. S-I flowsheet 2 equipment footprints.

\begin{tabular}{|c|c|c|c|c|}
\hline Flowsheet 2 & Item & Equipment Description & Area $\left(\mathrm{ft}^{2}\right)$ & Vol $\left(\mathrm{ft}^{3}\right)$ \\
\hline \multirow[t]{10}{*}{ Separators } & 129 & $\begin{array}{l}\text { Multi-stage isobaric flash vessel, Vertical Carbon Steel Vessel, } \\
5 \mathrm{ft} \text { dia. X } 33 \mathrm{ft} \text { tall }\end{array}$ & 5.0 & 165.0 \\
\hline & 130 & $\begin{array}{l}\text { Multi-stage isobaric flash vessel, Vertical Carbon Steel Vessel, } \\
5 \mathrm{ft} \text { dia. X } 33 \mathrm{ft} \text { tall }\end{array}$ & 5.0 & 165.0 \\
\hline & 131 & $\begin{array}{l}\text { Multi-stage isobaric flash vessel, Vertical Carbon Steel Vessel, } \\
5 \mathrm{ft} \text { dia. X } 33 \mathrm{ft} \text { tall }\end{array}$ & 5.0 & 165.0 \\
\hline & 132 & $\begin{array}{l}\text { Multi-stage isobaric flash vessel, Vertical Carbon Steel Vessel, } \\
5 \mathrm{ft} \text { dia. X } 33 \mathrm{ft} \text { tall }\end{array}$ & 5.0 & 165.0 \\
\hline & 133 & $\begin{array}{l}\text { Column Vessel, Carbon Steel Vertical Vessel, 12ft dia. X } 34.5 \\
\mathrm{ft} \text { tall, }\end{array}$ & 113.0 & 3899.9 \\
\hline & 134 & $\begin{array}{l}\text { Flash Separator, Carbon steel Vertical Vessel, } 5 \mathrm{ft} \text { dia. x } 25 \mathrm{ft} \\
\text { tall, }\end{array}$ & 19.6 & 490.6 \\
\hline & 135 & $\begin{array}{l}\text { Flash Separator, Carbon steel Vertical Vessel, } 4 \mathrm{ft} \text { dia. } \times 32 \mathrm{ft} \\
\text { long, }\end{array}$ & 12.6 & 401.9 \\
\hline & 136 & $\begin{array}{l}\text { Flash Separator, Carbon steel Vertical Vessel, } 4.5 \mathrm{ft} \text { dia. } \mathrm{x} \\
19.5 \mathrm{ft} \text { long, }\end{array}$ & 15.9 & 310.0 \\
\hline & 137 & $\begin{array}{l}\text { Column Partial Condenser Knockout, Carbon Steel, Horizontal } \\
\text { Vessel, } 4.5 \mathrm{ft} \text { dia. X } 19.5 \mathrm{ft} \text { long }\end{array}$ & 15.9 & 310.0 \\
\hline & 138 & $\begin{array}{l}\text { Flash Separator, Carbon steel, Vertical Vessel, } 4.5 \mathrm{ft} \text { dia. X } 10 \\
\mathrm{ft} \text { tall, }\end{array}$ & 15.9 & 159.0 \\
\hline \multirow[t]{18}{*}{ Heat Exchangers } & 139 & $\begin{array}{l}\text { Feed preheater/Product cooler, CS/Ni alloy Shell and tube, } \\
\text { fixed tube sheet heat exchanger }\end{array}$ & 835.0 & 40849.4 \\
\hline & $140-1$ & $\begin{array}{l}\text { Stage } 1 \text { of isobaric concentrator/product cooler, CS/SIC Shell } \\
\text { and tube, fixed tube sheet heat exchanger }\end{array}$ & 1815.0 & 130909.6 \\
\hline & $140-2$ & $\begin{array}{l}\text { Stage } 2 \text { Flash Heater/helium, CS/SIC Shell and tube, fixed } \\
\text { tube sheet heat exchanger }\end{array}$ & 2388.0 & 197564.0 \\
\hline & $140-3$ & $\begin{array}{l}\text { Stage } 3 \text { Flash Heater/helium, CS/SIC Shell and tube, fixed } \\
\text { tube sheet heat exchanger }\end{array}$ & 615.0 & 25820.8 \\
\hline & $140-4$ & $\begin{array}{l}\text { Stage } 4 \text { Flash Heater/helium, CS/SIC Shell and tube, fixed } \\
\text { tube sheet heat exchanger }\end{array}$ & 444.0 & 15839.1 \\
\hline & 141 & $\begin{array}{l}\text { Flash Cooler/Vaporizer preheater, CS/NI Shell and tube, Fixed } \\
\text { tube sheet heat exchanger }\end{array}$ & 5007.0 & 599822.6 \\
\hline & 142 & $\begin{array}{l}\text { Helium/2nd Vaporizer preheater, CS/NI Shell and tube, Fixed } \\
\text { tube sheet heat exchanger }\end{array}$ & 348.0 & 10990.7 \\
\hline & 143 & $\begin{array}{l}\text { Vaporizer, CS/SiC Shell and tube, fixed tube sheet heat } \\
\text { exchanger }\end{array}$ & 3641.0 & 371952.5 \\
\hline & 144 & $\begin{array}{l}\text { Recuperator, CS/SiC Shell and tube, fixed tube sheet heat } \\
\text { exchanger }\end{array}$ & 2395.4 & 198483.1 \\
\hline & 145 & Decomposer, Diffusion bonded Alloy $800 \mathrm{HT}$ PCHE & 2348.0 & 192621.0 \\
\hline & 146 & $\begin{array}{l}\text { Product Cooler/cooling water, CS/Ni, Shell and tube, fixed } \\
\text { tube sheet heat exchanger }\end{array}$ & 957.0 & 50121.5 \\
\hline & 147 & $\begin{array}{l}\text { Column Reboiler/Condensate cooler, CS/Ci Shell and tube, } \\
\text { fixed tube sheet heat exchanger. }\end{array}$ & 1019.4 & 55102.8 \\
\hline & 148 & $\begin{array}{l}\text { Condensate Cooler, CS/CS, Shell and tube, fixed tube sheet } \\
\text { heat exchanger }\end{array}$ & 250.0 & 6692.2 \\
\hline & 149 & $\begin{array}{l}\text { Condensate Cooler, CS/CS, Shell and tube, fixed tube sheet } \\
\text { heat exchanger }\end{array}$ & 370.0 & 12049.2 \\
\hline & 150 & $\begin{array}{l}\text { Partial Condenser for 2nd column feed, CS/SS Shell and tube } \\
\text { sheet heat exchanger }\end{array}$ & 1206.0 & 70905.1 \\
\hline & 151 & $\begin{array}{l}\text { Condensate Cooler, CS/CS, Shell and tube, fixed tube sheet } \\
\text { heat exchanger }\end{array}$ & 1681.0 & 116683.1 \\
\hline & 152 & $\begin{array}{l}\text { Cloumn Condenser, CS/Ci Shell and tube, fixed tube sheet } \\
\text { heat exchanger }\end{array}$ & 6312.0 & 848998.5 \\
\hline & 153 & $\begin{array}{l}\text { Feed preheater/helium, CS/Ni alloy Shell and tube, fixed tube } \\
\text { sheet heat exchanger }\end{array}$ & 2267.6 & 182812.6 \\
\hline Turbines & 154 & $\begin{array}{l}\text { Flash Water Expander,152 shaft hp, Carbon Steel, Liquid } \\
\text { Expander }\end{array}$ & 3.4 & 5.1 \\
\hline \multirow[t]{5}{*}{ Pumps } & 155 & Vaporizer feed pump, 329 shaft hp, Ni Alloy, Centrifugal Pump & 5.1 & 10.2 \\
\hline & 156 & Recycle Pump, 688 shaft hp, Epoxy lined, Centrifugal pump & 9.4 & 31.1 \\
\hline & 157 & Water pump, 11 shaft hp, Epoxy lined, Centrifugal Pump & 1.3 & 0.9 \\
\hline & 158 & Feed Pump, 1738 shaft hp, Plastic lined, Centrifugal Pump & 15.7 & 47.0 \\
\hline & & Total for Equipment & 34,147 & $3,134,543$ \\
\hline
\end{tabular}




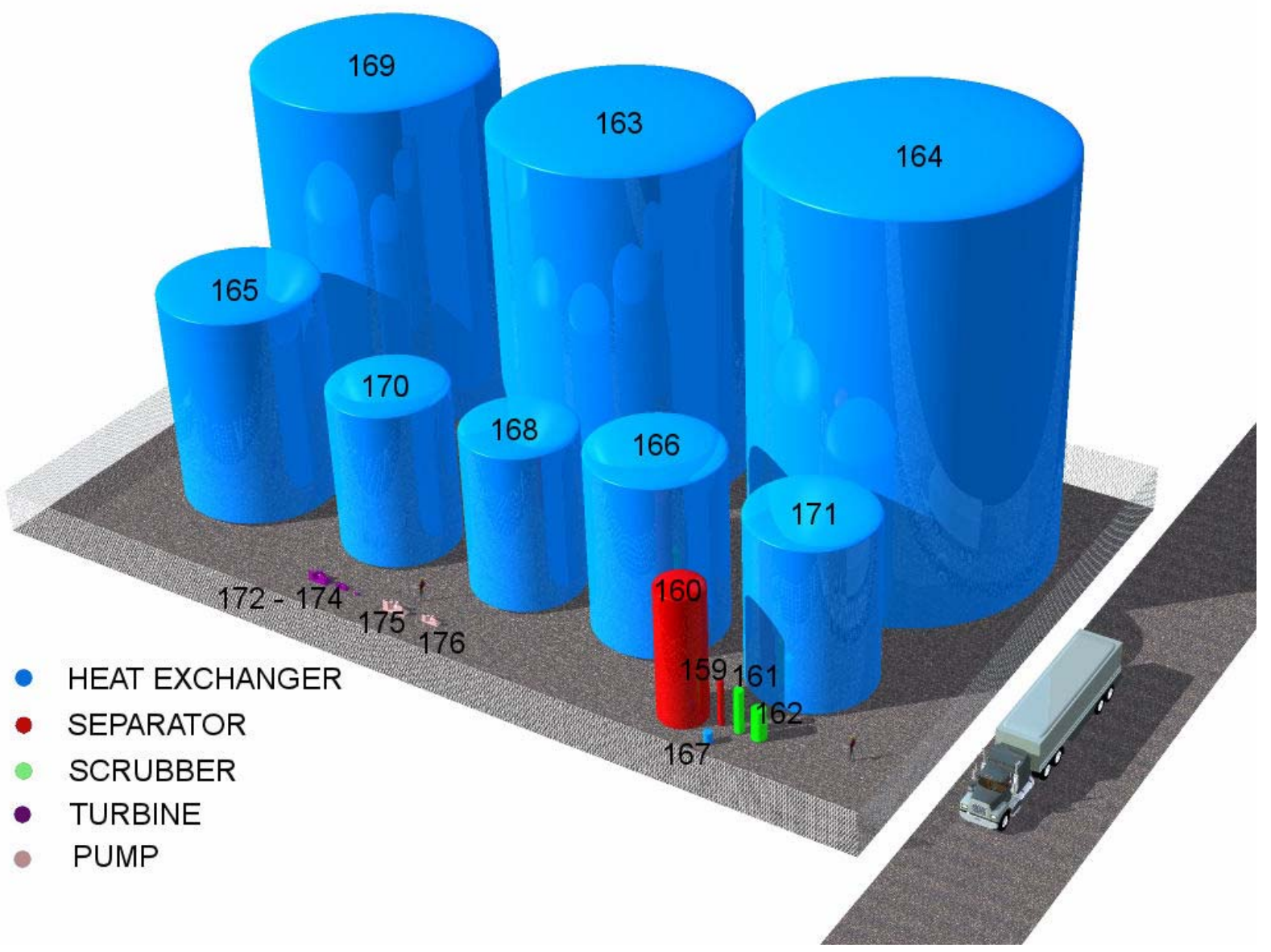

Figure 9. S-I flowsheet 3 facility 


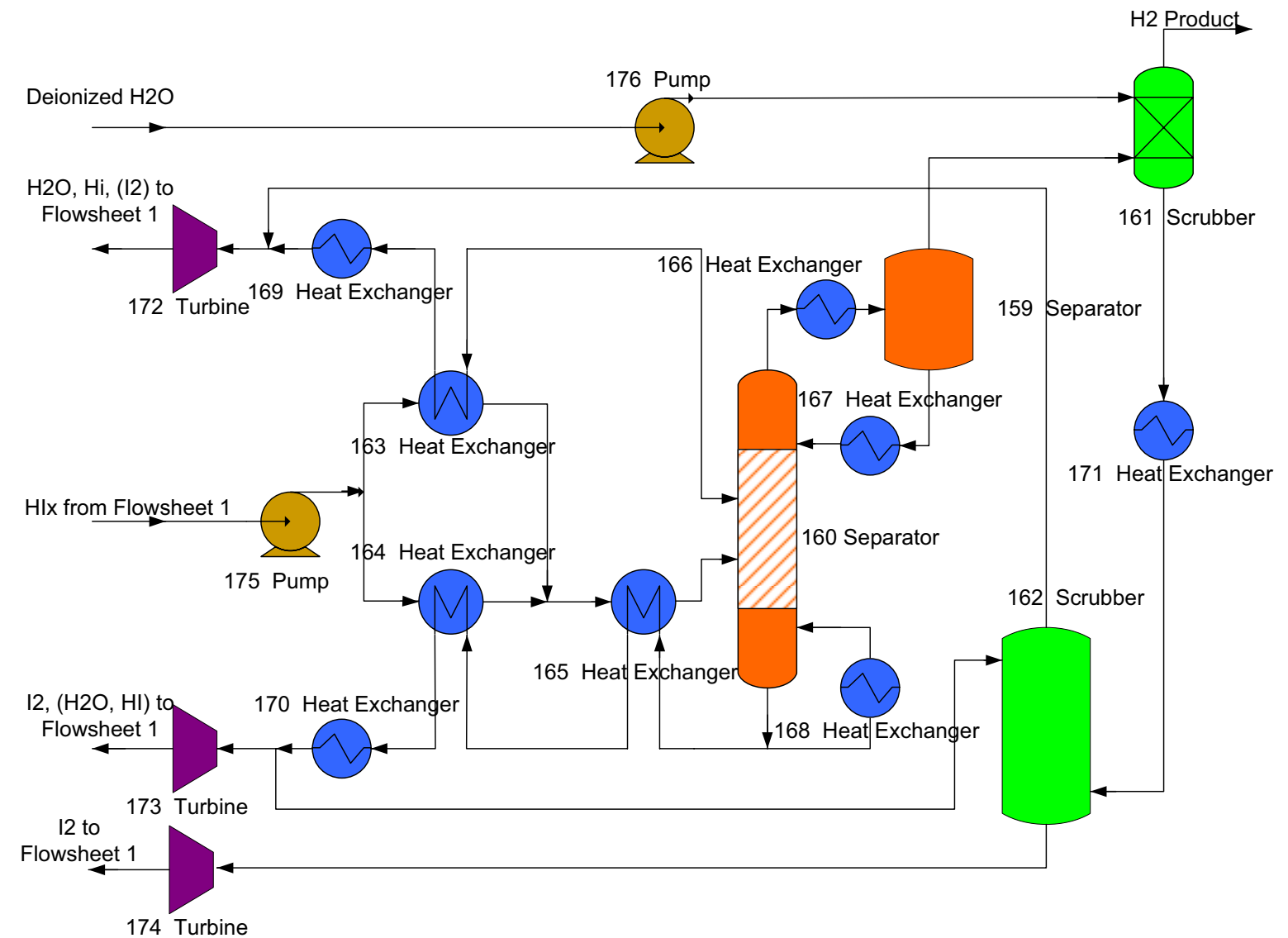

Figure 10. S-I flowsheet 3 
Table 5. S-I flowsheet 3 equipment footprints.

\begin{tabular}{|c|c|c|c|c|}
\hline Flowsheet 3 & Item & Equipment Description & Area $\left(\mathrm{ft}^{2}\right)$ & Vol $\left(\mathrm{ft}^{3}\right)$ \\
\hline \multirow[t]{2}{*}{ Separators } & 159 & $\begin{array}{l}\text { Condenser Drum, Vertical Vessel, } 1.25 \mathrm{ft} \text { dia. X } 10.5 \mathrm{ft} \text { tall, } \\
\text { Carbon Steel }\end{array}$ & 1.2 & 12.9 \\
\hline & 160 & Reactive distillation column, $10 \mathrm{ft}$ dia. $\times 31 \mathrm{ft}$ tall, Carbon Steel & 78.5 & 2433.5 \\
\hline \multirow[t]{2}{*}{ Scrubbers } & 161 & H2 Scrubber, Vertical Vessel, $2 \mathrm{ft}$ dia. $X 10 \mathrm{ft}$ tall, Carbon Steel & 3.1 & 31.4 \\
\hline & 162 & $\begin{array}{l}\text { I2 Scrubber for Sec. I Boost Reactor, Vertical Vessel, } 3 \mathrm{ft} \text { dia. } \\
\times 7 \mathrm{ft} \text { tall, Carbon steel }\end{array}$ & 7.1 & 49.5 \\
\hline \multirow[t]{9}{*}{ Heat Exchangers } & 163 & $\begin{array}{l}\text { Recuperator, Shell and tube, fixed tube sheet heat exchanger, } \\
\text { CS/CS }\end{array}$ & 2348.0 & 192621.0 \\
\hline & 164 & $\begin{array}{l}\text { Recuperator, Shell and tube, fixed tube sheet heat exchanger, } \\
\text { CS/CS }\end{array}$ & 3074.0 & 288544.1 \\
\hline & 165 & $\begin{array}{l}\text { Recuperator, Shell and tube, fixed tube sheet heat exchanger, } \\
\text { CS/CS }\end{array}$ & 917.0 & 47012.2 \\
\hline & 166 & $\begin{array}{l}\text { Condenser, Shell and tube, fixed tube sheet heat exchanger, } \\
\text { CS/Karbate }\end{array}$ & 604.0 & 25131.1 \\
\hline & 167 & $\begin{array}{l}\text { Condensate Reheater, Shell and tube, fixed tube sheet heat } \\
\text { exchanger, CS/SiC }\end{array}$ & 2.5 & 6.7 \\
\hline & 168 & $\begin{array}{l}\text { Reboiler, Shell and tube, fixed tube sheet heat exchanger, } \\
\text { CS/SiC }\end{array}$ & 451.0 & 16215.2 \\
\hline & 169 & $\begin{array}{l}\text { Water Recycle heat exchanger, Shell and tube, fixed tube } \\
\text { sheet heat exchanger, CS/Karbate }\end{array}$ & 2231.0 & 178404.5 \\
\hline & 170 & $\begin{array}{l}\text { lodine Recycle heat exchanger to Sec. I, Shell and tube, fixed } \\
\text { tube sheet heat exchanger, CS/Karbate }\end{array}$ & 509.0 & 19441.6 \\
\hline & 171 & $\begin{array}{l}\text { Reboiler, Shell and tube, fixed tube sheet heat exchanger, } \\
\text { CS/SiC }\end{array}$ & 528.0 & 20540.3 \\
\hline \multirow[t]{3}{*}{\begin{tabular}{|l|} 
Turbines \\
\end{tabular}} & 172 & Recycle Water Liquid Expander, 695 shaft hp, Ni Alloy & 7.6 & 15.2 \\
\hline & 173 & Recycle lodine Liquid Expander, 1197 shaft hp, Ni Alloy & 12.5 & 28.3 \\
\hline & 174 & $\begin{array}{l}\text { lodine to Sec. I Boost reactor liquid expander, } 203 \text { shaft hp, Ni } \\
\text { Alloy }\end{array}$ & 4.1 & 6.1 \\
\hline \multirow[t]{3}{*}{ Pumps } & 175 & Feed Centrifugal Pump, 462 shaft hp, Stainless Steel & 6.6 & 13.2 \\
\hline & 176 & De-lonized Centrifugal Pump, 180 shaft Hp, Stainless Steel & 2.8 & 4.2 \\
\hline & & Total for Equipment & 10,788 & 790,511 \\
\hline
\end{tabular}




\subsection{High Temperature Electrolysis (HTE)}

High Temperature Electrolysis (HTE) uses both thermal and electrical energy to split water directly into hydrogen and oxygen using an electrolytic cell operating at $750-900{ }^{\circ} \mathrm{C}$. A mixture of steam and hydrogen having a 9-to-1 volume ratio enters the anode of the cell. At the anode surface, water decomposes into oxygen and hydrogen ions. Hydrogen gas is formed at the anode from the recombination of hydrogen ions into hydrogen gas. The potential difference across the cell and the cell's unique membrane structure motivates oxygen ions to diffuse across the cell membrane to the cathode, where the oxygen ions recombine to form oxygen gas. Hydrogen gas is separated from the gas stream exiting the anode, while oxygen is removed from the cell as a pure gas. Process requirements were estimated utilizing the HYSYS model reported by Carl Stoots, James O'Brien, Mike McKellar, Grant Hawkes, and Stephen Herring in an internal INEEL report published in June 2004.

Table 6. HTE process requirements

\begin{tabular}{|c|c|}
\hline High Temperature Electrolysis & $600 \mathrm{MW}$ \\
\hline Heating Load (either electrical or fossil) & $102 \mathrm{MW}$ \\
\hline Power for Electrolysis and Prime Movers & $600 \mathrm{MW}$ \\
\hline \multicolumn{2}{|l|}{ Process Space Requirement } \\
\hline No. of Vessels & 16 \\
\hline No. of Processing Sections & 1 \\
\hline Vessel Footprint & $23,790 \mathrm{ft}^{2}$ \\
\hline Plant Footprint (based on 10:1 ratio) & $237,900 \mathrm{ft}^{2}$ \\
\hline Access Area (based on 3:4 plant footprint ratio) & $178,425 \mathrm{ft}^{2}$ \\
\hline Control System (based on 1:8 plant footprint ratio) & $29,738 \mathrm{ft}^{2}$ \\
\hline Total Space Requirements & $446,063 \mathrm{ft}^{2}$ \\
\hline
\end{tabular}




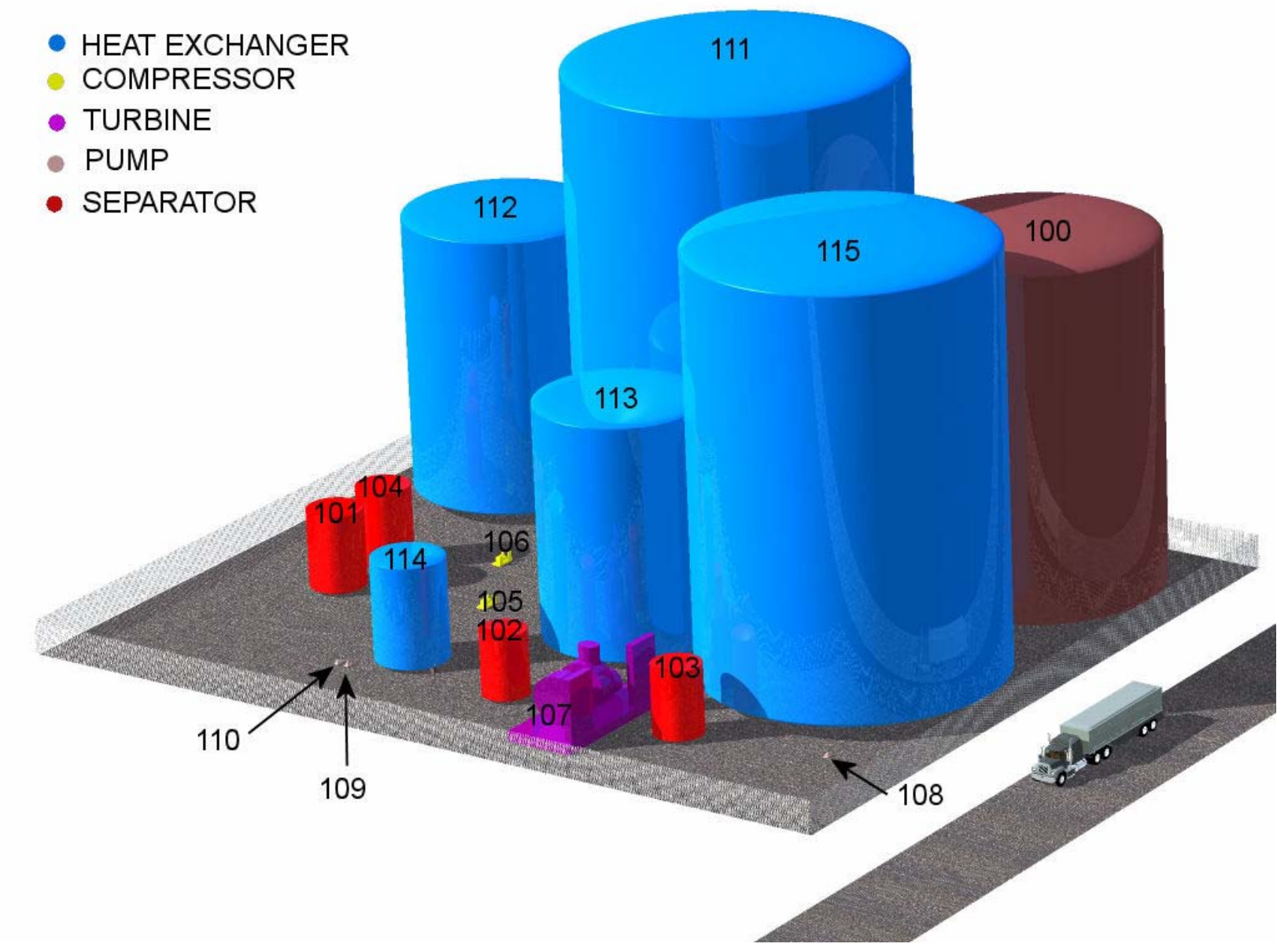

Figure 11. HTE flowsheet facility. 


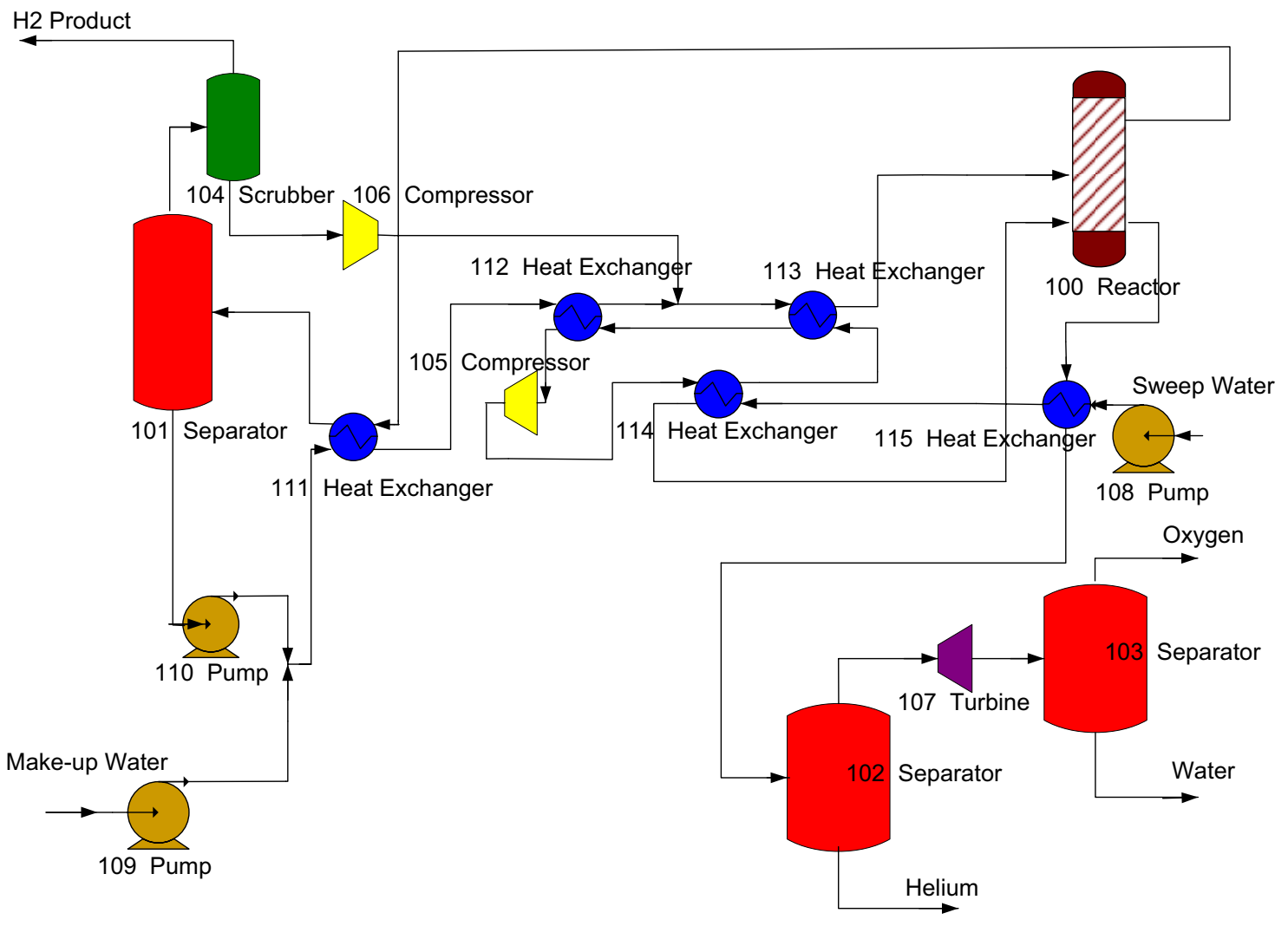

Figure 12. HTE flowsheet.

Table 7. HTE equipment footprints

\begin{tabular}{|c|c|c|c|c|}
\hline Flowsheet & Item & Equipment Description & Area $\left(\mathrm{ft}^{2}\right)$ & Vol $\left(\mathrm{ft}^{3}\right)$ \\
\hline Reactors & 100 & Electroylzer, Vertical vessel, $72 \mathrm{ft}$ dia X $108 \mathrm{ft}$ tall, $\mathrm{CS} / \mathrm{Ni}$ & 4050.0 & 436354.0 \\
\hline \multirow[t]{3}{*}{ Separators } & 101 & H2/Water Knockout Tank, Vertical Carbon Steel Vessel, $16 \mathrm{ft} \mathrm{dia} \mathrm{X} 24 \mathrm{ft}$ tall & 208.0 & 5120.0 \\
\hline & 102 & $\begin{array}{l}\begin{array}{l}\text { High Pressure H20/02 Knockout Tank, Vertical Carbon Steel Vessel, } 13 \mathrm{ft} \text { dia X } 20 \mathrm{ft} \\
\text { tall }\end{array} \\
\end{array}$ & 140.7 & 2847.0 \\
\hline & 103 & Low Pressure H20/02 knockout Tank, Vertical Carbon Steel Vessel, $14 \mathrm{ft} \mathrm{dia} \times 21 \mathrm{ft}$ tall & 158.8 & 3414.0 \\
\hline Scrubbers & 104 & H2 Scrubber, Vertical Carbon Steel Vessel, $16 \mathrm{ft}$ dia X $24 \mathrm{ft}$ tall & 199.3 & 4802.0 \\
\hline \multirow[t]{2}{*}{ Compressors } & 105 & Compressor, $1550 \mathrm{hp}$, Ni Alloy & 16.8 & 48.7 \\
\hline & 106 & H2 Compressor, $20 \mathrm{hp}$, Ni Alloy & 1.8 & 2.2 \\
\hline Turbines & 107 & O2/Steam Turbine, $11200 \mathrm{hp}, \mathrm{Ni}$ Alloy & 876.6 & 18362.8 \\
\hline \multirow[t]{3}{*}{ Pumps } & 108 & Fluorocarbon lined Centrifugal pump, 93 shaft $\mathrm{hp}$ & 2.4 & 3.0 \\
\hline & 109 & Fluorocarbon lined Centrifugal pump, 189 shaft $\mathrm{hp}$ & 3.1 & 4.9 \\
\hline & 110 & Fluorocarbon lined Centrifugal pump, 1.2 shaft $\mathrm{hp}$ & 1.0 & 0.5 \\
\hline \multirow[t]{6}{*}{ Heat Exchangers } & 111 & Recuperator, CS/SiC Shell and tube, fixed tube sheet heat exchanger & 7441.0 & 1086684.3 \\
\hline & 112 & Recuperator, CS/SiC Shell and tube, fixed tube sheet heat exchanger & 2663.0 & 232655.4 \\
\hline & 113 & Recuperator, CS/SiC Shell and tube, fixed tube sheet heat exchanger & 1836.0 & 133188.1 \\
\hline & 114 & Recuperator, CS/SiC Shell and tube, fixed tube sheet heat exchanger & 349.0 & 11038.1 \\
\hline & 115 & Recuperator, CS/SiC Shell and tube, fixed tube sheet heat exchanger & 5843.0 & 756154.0 \\
\hline & & Total for Equipment & 23,790 & $2,690,679$ \\
\hline
\end{tabular}




\section{GENERAL BOP REQUIREMENTS}

This section describes BOP requirements that apply to the hydrogen production plant in general, outside of the chosen hydrogen production technology.

\subsection{Fluid Motivators}

Fluid motivators are devices for moving fluids from one location to another. They may be pumps, compressors, ejectors, or other such devices. Such devices will be needed to move fluids between process units, collect waste and product streams, and to fill and drain process units during start-up and shutdown activities. Fluid motivator technologies must be carefully matched to the fluids of interest to achieve maximum energy efficiency.

\subsection{Heat Recovery and Heat Rejection}

The BOP must include equipment and operating methods for recovering higher quality (higher temperature) thermal energy from process flow streams and for rejecting low quality (lower temperature) thermal energy from the process. Heat recovery is necessary to approach the high theoretical energy efficiencies offered by the candidate hydrogen production processes.

The hydrogen generation process involves flow streams and reaction vessels operating in a temperature range from ambient (inlet feed temperatures) to a maximum of $850-900{ }^{\circ} \mathrm{C}$ (sulfur-iodine cycle). Heat recovery involves the use of stored thermal energy or the thermal capacity in the process fluids to heat or cool process flow streams for the purposes of recovering heat energy and minimizing the need for external heating and cooling. Heat recovery contributes to overall process energy efficiency. Heat recovery can be performed using heat exchangers, collocated pipes, and other such devices. Design and modeling of heat recovery equipment must be optimized through pinch point analysis and other methods to make best use of available thermal energy resources.

In the sulfur-iodine cycle, for example, the $\mathrm{H}_{2} \mathrm{SO}_{4}$ generated at a lower temperature by the Bunsen reaction must be heated to $850{ }^{\circ} \mathrm{C}$ in order to undergo the decomposition reaction into $\mathrm{SO}_{2}, \mathrm{O}_{2}$, and $\mathrm{H}_{2} \mathrm{O}$. Thermal energy recovered from process streams can be used to at least partially heat the sulfuric acid to operating temperature so that less external heating is needed. Such heating of input streams can be used effectively in the other hydrogen production processes, as well.

Eventually, exiting process streams have been cooled to the point where no more useful process heat can be withdrawn from them. In some cases, however, the temperature of the exit stream may still be too high to allow the stream contents to be stored or vented. Under such circumstances, equipment such as air- or water-cooled heat exchangers may be needed to lower the temperature of those streams so the stream contents can be safely handled or stored. Rejected thermal energy may be vented directly into the environment or used to heat rooms and buildings.

\subsection{Control Systems}

Control systems are needed to monitor and maintain system temperatures, pressures, and flow rates at the desired levels. Their design should incorporate audible and visual alarms to allow operator intervention or to take automatic action if measurements indicate that the process is operating outside of normal operating conditions. The alarm indicators should signal both locally, and at the hydrogen production plant's central control station. Control systems should be integrated so that the responses of individual controllers are in harmony with the responses of other system controllers and are not in opposition. The specific types of control systems needed are described in the following sections. 


\subsubsection{Temperature Control}

Process vessels, pipes, heat exchangers, and other equipment in the hydrogen generation process all operate at different temperatures. To maintain the proper operating temperature for each process unit, temperature monitoring and temperature control will be needed. Temperature monitoring is performed by temperature sensors (e.g., thermocouples, thermistors, etc.), and temperature control is performed by controlling units that alter the input and output flow rates and the power to direct heating/cooling units to maintain temperature set points. Optimizing the placement of temperature sensors (e.g., thermocouples) will minimize the number of temperature sensors required by the temperature controller. Depending upon the design of the temperature controller, the temperature of a single component may be monitored and controlled, or the results from many temperature sensors may be integrated and analyzed in order to control the temperature of a whole process unit, or even influence the overall operation of the hydrogen production plant.

\subsubsection{Flow Control}

Material transport between process units requires flow monitoring and control to keep the process steps operating at optimum conditions. Depending upon the process, flow monitoring and control can vary in design and function. Similar to temperature control, flow monitoring sensors (e.g., mass flow meters, velocimeters, etc.) provide input to one or more flow controllers. Flow controllers influence the flow rates by changing the position of flow control valves and/or adjusting the work rates of pumps or compressors. Flow control can be local and focused on only one component, or the signals from many flow sensors can be integrated to control the flow rates in many components or locations simultaneously.

\subsubsection{Pressure Control}

Though temperature and flow control is often sufficient to maintain system pressures, in some cases additional pressure protection may be required, as realized through pressure control. A pressure controller will receive input from one or more pressure sensors in a unit or flow stream, and then motivate a response in order to raise or lower the measured pressure so that it falls within its operating bounds. This response may be to influence other temperature or pressure controllers to change system temperatures or flow rates, or it may be to manipulate valves, pumps, and other equipment directly in order to affect the measured pressures. Pressure protection for vessels that operate at elevated pressures must be evaluated to determine whether passive pressure protective devices are sufficient, or whether active pressure control systems are needed.

\subsubsection{Other Controllers}

Depending upon the process specifics, other controllers may also be needed, such as liquid level controllers, compositional controllers, and so forth. The need for such controllers will be evaluated during the plant design and development process.

\subsection{Safety Systems}

Safety systems, depending upon their function, protect personnel and equipment. The BOP must contain both manually operated safety equipment (e.g., fire extinguishers) and automatic safety systems, as appropriate for the specific hazards that may be encountered in the hydrogen production plant. The facility design provides some inherent safety protection by virtue of its physical structure and operating control systems, but additional safety systems will be required to protect personnel and equipment in the unlikely event of control system failures, fluid leaks, fires, and other such problems. Depending upon the degree of protection desired, automatic safety systems can be tied in with the plant control systems so that the plant's temperature, flow, and pressure controllers can place the plant into a safer condition automatically in the event of a safety problem. Before start-up and periodically during regular operation, 
regular safety audits and safety inspections should be performed in order to ensure that all safety equipment and safety systems are in place and operational.

\subsubsection{Manual Safety Systems}

BOP must include placement of manual safety systems such as fire extinguishers, safety showers, eye wash stations, and other such equipment to protect personnel. Fire alarm pull boxes must be placed along worker access routes as needed to allow quick notification of fire and safety personnel. The facility must be adequately lighted and well marked with safety signs. Walkways and access areas must be demarcated by pathways, catwalks, and other such devices, and hazardous areas must be marked accordingly. Manual safety systems may also include the use of hand-held radio systems, "man-down" monitors, and other such equipment that workers may carry with them in the course of their duties.

\subsubsection{Automatic Safety Systems}

Automatic safety systems are systems that do not require human intervention before activating. These systems include automatic pressure relief valves, fire detection systems, alarm systems, automatic fire control systems, and other such devices. Automatic safety systems are appropriate when the response time of the safety system must be rapid and/or the safety system receives inputs from process sensors and control systems. It is desirable, but not required, to maximize the use of automatic safety systems so that the risk to workers in hazard response situations can be minimized.

\subsection{Water Purification}

The hydrogen production processes require pure water. Since the S-I process is cyclic, and since the HTE process relies on the use of membranes, it will be necessary to provide the purest water for a feed stream so that water contaminants do not build up in process equipment. Water purification equipment, including a water distillation column or boiler, may be needed to purify the water feed stream prior to injecting it into the process.

If the water source for the hydrogen plant is salt water, water desalinization equipment may also be needed to purify the water stream.

\subsection{Product Recovery}

The two major products from the hydrogen plant will be hydrogen and oxygen. Both are useful products and must be recovered for use. Since both of these products are a gas at atmospheric conditions, these materials will need to be compressed and/or liquefied for storage or transport to a user.

Compressors and/or liquefaction equipment and product storage containers (or pipelines) must be installed in the plant to collect the hydrogen and oxygen produced by the plant.

\subsection{Non-Product Stream Treatment and Disposal}

In the course of operation, almost all chemical plants will generate material streams that are not the main products of the plant. These extra material streams may be gaseous, liquid, or solid. In theory, there will be no material streams other than hydrogen and oxygen. In practice, there may be other streams due to impure separations, unwanted side reactions, the build-up of corrosion products in recycle loops, and other such events. Material streams may also be created by leaks, spills, and other non-routine events.

These other material streams require support equipment to collect or treat them. Such support equipment may take the form of line filters, absorption beds, catch tanks, by-product collection equipment, or other such mass collection devices, as appropriate for the particular material stream. For 
oxygen or hydrogen streams, compressors and/or liquefaction equipment may be needed to collect and store the product streams. Catch basins and other such devices, otherwise classified as "secondary containment," will be needed under all process vessels and containers to collect process liquid wastes that may leak out in the case of spills or vessel rupture. The catch basin itself, or a linked collection vessel, must have sufficient volume to contain the entire volume of the vessel it protects. The need for material collection devices for these extra material streams will be evaluated during the hydrogen plant design phase, so that they can be included in the plant design.

If the potential exists to vent pollutants regulated by the Environmental Protection Agency (EPA) or other regulating bodies, then it will be necessary to install pollution control devices to limit the emission of such waste products into the environment.

\subsection{Heating, Ventilation, and Air Conditioning (HVAC)}

In enclosed spaces where people must work, it may be necessary to install heating, ventilation and air conditioning (HVAC) in order to provide a comfortable and safe place to work. The hydrogen production processes all require the use of very high temperatures and may require the use of hazardous solvents. Without appropriate HVAC systems, work spaces may become hazardous due to heat, cold, or pollutants. HVAC systems will help protect enclosed spaces so that workers may work in those areas without the use of ventilators and other such cumbersome protective equipment. In enclosed portions of the plant that require worker access for maintenance or repair, HVAC systems must be provided to protect the worker space.

\subsection{Electrical Distribution}

The hydrogen production plant equipment will require electrical power at various voltages and phases. Though it is expected that the plant infrastructure will supply all of the electricity needed by the plant, electrical equipment within the plant will distribute that electricity at the proper voltages and power levels to each plant system, according to each system's needs. Electrical distribution equipment includes such devices as transformers, rectifiers, circuit breakers, back-up power systems, surge suppressors, and other such equipment. Emergency lighting using a back-up power source will also be needed to provide illumination in the event that primary electrical power to the plant is lost.

\subsection{Storage Vessels}

Chemical storage vessels will be an integral part of the hydrogen plant's support systems. Water storage tanks will be needed to supply water to the hydrogen production process. Product storage tanks will be needed to store the hydrogen and oxygen produced.

To facilitate plant start-up, shutdown, and vessel maintenance, vessel dump tanks will be needed to store the contents of vessels, so that the vessels can be filled and drained with process chemicals. Vessel dump tanks should be of sufficient size to hold the entire volume of fluid within its corresponding process vessel.

In processes using multiple processing steps, temporary storage tanks may be needed as a buffer between process units to level out feed flow rates. The buffer tanks do not need to be the same size as the vessel they correspond to, but they need to be large enough to perform their function, as determined by process models and laboratory testing results.

Waste storage vessels may also be required, especially if recycle loops employ purge streams to remove contaminants. Depending upon the purge stream, the stream contents may be held for reprocessing, or it may be stored for eventual disposal. In any case, it is likely that the flow rate of the 
purge stream will be small enough that immediate stream treatment activities will not be performed at the time of stream withdraw, thus storage vessels will be needed.

As part of a detailed design process, the need for storage vessels and the sizing of storage vessels at various parts of the hydrogen production process must be evaluated, so that sufficient storage is allotted in the plant design.

\subsection{Maintainability}

The BOP must be constructed with plant maintainability as a design parameter. With high operating temperatures and reactive chemicals, the hydrogen plants are likely to require frequent maintenance to maintain operability. To facilitate maintenance activities, piping, pumps, vessels, and other equipment must be accessible to workers. Parts must be repairable or easily replaced without having to disassemble the hydrogen plant. Vessels having large volumes must be isolatable and drainable, so that the particular part can be isolated from the rest of the plant and then drained of process fluids when necessary.

The plant should also be equipped with sample insertion points at which material samples can be exposed to process fluids. Materials corrosion will be a large concern in the hydrogen plant, and the search for materials that better resist corrosive environments will be an ongoing activity. It will be invaluable to be able to test material samples within the actual hydrogen plant fluid streams, so that material samples can be exposed to actual process conditions. Placing sample insertion locations throughout the plant will expand materials knowledge databases and will facilitate future improvements in plant equipment reliability.

\section{PROCESS-SPECIFIC BOP REQUIREMENTS}

In addition to the BOP requirements that apply generically to the hydrogen production plant, certain other BOP requirements will apply, depending upon the chosen hydrogen production method. This section describes the additional BOP requirements that are relevant to specific hydrogen production technologies.

\subsection{Catalyst Regeneration for S-I}

The decomposition of $\mathrm{H}_{2} \mathrm{SO}_{4}$ into $\mathrm{SO}_{2}, \mathrm{H}_{2} \mathrm{O}$ and $\mathrm{O}_{2}$ in the $\mathrm{S}$-I process is catalyzed, usually by a vanadium-based catalyst. The catalyst, by virtue of its chemical composition and surface microstructure, lowers the activation energy of the conversion reaction between $\mathrm{SO}_{3}$ and $\mathrm{SO}_{2}$ and, thereby, increases the reaction rate. Over time, the catalyst surface can become clogged with reaction by-products, or the catalyst itself may undergo some chemical degradation, so that it becomes less effective. Once the catalyst becomes degraded, it will be necessary to regenerate or replace the catalyst.

S-I hydrogen production plants must have support equipment available to facilitate catalyst regeneration and/or catalyst replacement in the $\mathrm{H}_{2} \mathrm{SO}_{4}$ decomposition reactor.

\subsection{Corrosion Product Removal in S-I Process}

The S-I and SE processes both use $\mathrm{H}_{2} \mathrm{SO}_{4}$ and $\mathrm{H}_{2} \mathrm{O}$ in their process loops. The S-I process also uses $\mathrm{I}_{2}$ and $\mathrm{HI}$. At the temperatures used by these two processes, the listed chemicals are all highly corrosive to structural materials. Even with corrosion-resistant materials, it is likely that some components will degrade over time due to corrosion. 
In the liquid process streams, it is likely that corrosion products will become dissolved or entrained. With no material outlet for these particles, due to the cyclical nature of these processes, it is possible for the concentration of corrosion particles to build up to disruptive levels. Equipment must be placed on recycle lines (purge valves, filters, ion-exchange columns, absorbers, etc.) to remove these corrosion products.

\subsection{Product Purification in S-I Process}

In the S-I process, it is possible to contaminate the hydrogen product stream with halogens and/or sulfur compounds if the base product separation processes are not operating perfectly. In the S-I process, hydrogen may become contaminated with $\mathrm{SO}_{2}$, $\mathrm{HI}$, or $\mathrm{I}_{2}$ vapor.

In any case, the hydrogen product stream must be made as pure as possible so that the hydrogen can be employed for use in fuel cells without damaging fuel cell membranes and hydrogen storage medium. If leak-through of by-products is witnessed during initial laboratory testing, then it may be necessary to install additional hydrogen purification equipment to further clean-up the hydrogen product stream.

Also, in these processes, the oxygen product stream may become contaminated with sulfur and/or halogen compounds. If such contamination is witnessed during initial laboratory testing, it will be necessary to install oxygen purifying equipment in the plant, as well.

\subsection{Hydrogen Purification in the HTE Process}

The high temperature electrolysis process uses only water as a reactant, and produces only hydrogen and oxygen as products. Oxygen, being produced at the cathode of the electrochemical cells, emerges as a pure gas. Hydrogen, produced at the anode, emerges from the electrochemical cell in a mixture of hydrogen gas and water vapor. The hydrogen gas must be separated from the water vapor to produce a pure hydrogen stream. Membranes, heat exchanger units, and other such devices may be needed to perform this purification process.

\section{SUMMARY OF REQUIREMENTS}

BOP requirements described in this document provide a list of systems and equipment that will be needed to support the hydrogen generation chemical reactions in a nuclear hydrogen production plant. Although the list of requirements is somewhat generic, a more refined list of requirements can be developed once details of the particular candidate hydrogen production process - S-I and HTE-have been fully defined. A summary of the BOP requirements described in this document is presented in Table 5. 
Table 8. Summary of Requirements

\begin{tabular}{|c|c|}
\hline \multicolumn{2}{|r|}{ Generic Requirements } \\
\hline Fluid Motivators & $\begin{array}{l}\text { - Pumps, compressors, ejectors, and other fluid motivators will } \\
\text { be needed to move process fluids throughout the plant. }\end{array}$ \\
\hline $\begin{array}{l}\text { Heat Recovery and Heat } \\
\text { Rejection }\end{array}$ & $\begin{array}{l}\text { - Thermal energy recovery, thermal energy integration, and } \\
\text { low-temperature heat rejection will be needed to improve } \\
\text { plant energy efficiency. Plant designs must include thermal } \\
\text { recovery designs and equipment. }\end{array}$ \\
\hline Control Systems & $\begin{array}{l}\text { - Temperature controllers. } \\
\text { - Flow controllers. } \\
\text { - Pressure controllers. } \\
\text { - Other controllers (liquid level, composition, etc.). }\end{array}$ \\
\hline Safety Systems & $\begin{array}{l}\text { - Automatic safety systems. } \\
\text { - Manually operated safety systems. }\end{array}$ \\
\hline Water Purification & - $\quad$ Purified water needed as process feed material. \\
\hline Product Recovery & $\begin{array}{l}\text { - Gas compression or liquefaction equipment needed for } \\
\text { product storage and delivery. }\end{array}$ \\
\hline $\begin{array}{l}\text { Non-Product Stream } \\
\text { Treatment and Disposal }\end{array}$ & $\begin{array}{l}\text { - Pollutant and waste treatment and/or disposal equipment } \\
\text { needed. }\end{array}$ \\
\hline HVAC & - HVAC needed in enclosed spaces. \\
\hline Electrical Distribution & $\begin{array}{l}\text { - Electrical power systems are needed to distribute electricity to } \\
\text { plant components at proper voltages and power levels. } \\
\text { - } \quad \text { Back-up power sources, surge suppression equipment. }\end{array}$ \\
\hline Storage Vessels & $\begin{array}{l}\text { - } \quad \text { Feed storage tank(s). } \\
\text { - Vessel and line dump tanks. } \\
\text { - Intermediate tanks for process control purposes. } \\
\text { - Waste collection tanks. }\end{array}$ \\
\hline Maintainability & - $\quad$ Plant maintainability must be a design parameter. \\
\hline \multicolumn{2}{|r|}{ Process-Specific Requirements } \\
\hline S-I Cycle & $\begin{array}{l}\text { - Catalyst regeneration equipment needed for } \mathrm{H}_{2} \mathrm{SO}_{4} \\
\text { - } \text { decomposition catalyst. } \\
\text { - } \quad \text { Product purification needed to remove trace halogens and } \\
\text { sulfur compounds. }\end{array}$ \\
\hline $\begin{array}{l}\text { High Temperature } \\
\text { Electrolysis }\end{array}$ & - $\mathrm{H}_{2}$ purification $\left(\mathrm{O}_{2}\right.$ stream already pure $)$ \\
\hline
\end{tabular}




\section{REFERENCES}

1. DOE Hydrogen Posture Plan, February 2004

2. Nuclear Hydrogen R\&D Plan, draft February 2004

3. General Atomics Report GA-A24285, June 2003

4. Carl M. Stoots, James E. O’Brien, Mike G. McKellar, Grant L. Hawkes, and J. Stephen Herring, Engineering Process Model for High-Temperature Electrolysis System

Performance Evaluation Internal Report, May, 2005.

5. J. Stephen Herring, James E. O’Brien, Carl M. Stoots, Paul A. Lessing, Hydrogen Production from Nuclear Energy via High-Temperature Electrolysis, presented at ICAPP '04, June 13-17, 2004, Pittsburgh, PA 